\title{
Functional loss of a noncanonical BCOR- PRC1.1 complex accelerates SHH-driven medulloblastoma formation
}

\author{
Lena M. Kutscher, ${ }^{1,2,16}$ Konstantin Okonechnikov, ${ }^{1,2,16}$ Nadja V. Batora, ${ }^{1,2,16}$ Jessica Clark, ${ }^{1,2}$ \\ Patricia B.G. Silva, ${ }^{1,2}$ Mikaella Vouri, ${ }^{1,2}$ Sjoerd van Rijn,${ }^{1,2}$ Laura Sieber, ${ }^{1,2}$ Britta Statz, ${ }^{1,2}$ \\ Micah D. Gearhart, ${ }^{3}$ Ryo Shiraishi ${ }^{4}$ Norman Mack, ${ }^{1,2}$ Brent A. Orr, ${ }^{5}$ Andrey Korshunov, ${ }^{6,7}$ \\ Brian L. Gudenas, ${ }^{8}$ Kyle S. Smith, ${ }^{8}$ Audrey L. Mercier, ${ }^{9,10}$ Olivier Ayrault, ${ }^{9,10}$ Mikio Hoshino, ${ }^{4}$ \\ Marcel Kool, ${ }^{1,2,11}$ Katja von Hoff, ${ }^{12}{ }^{12}$ Norbert Graf, ${ }^{13}$ Gudrun Fleischhack, $^{14}$ Vivian J. Bardwell, $^{3}$ \\ Stefan M. Pfister, ${ }^{1,2,15}$ Paul A. Northcott, ${ }^{8}$ and Daisuke Kawauchi ${ }^{1,2,4}$
}

\begin{abstract}
${ }^{1}$ Hopp-Children's Cancer Center Heidelberg (KiTZ), 69120 Heidelberg, Germany; ${ }^{2}$ Division of Pediatric Neurooncology, German Cancer Research Center (DKFZ), German Cancer Consortium (DKTK), 69120 Heidelberg, Germany; ${ }^{3}$ Department of Genetics, Cell Biology, and Development, Masonic Cancer Center, Developmental Biology Center, University of Minnesota, Minneapolis, Minnesota 55455, USA; ${ }^{4}$ Department of Biochemistry and Cellular Biology, National Institute of Neuroscience, National Center of Neurology and Psychiatry (NCNP), Tokyo 187-0031, Japan; ${ }^{5}$ Department of Pathology, St. Jude Children's Research Hospital, Memphis, Tennessee 38105, USA; ${ }^{6}$ Clinical Cooperation Unit Neuropathology, German Cancer Research Center (DKFZ), 69120 Heidelberg, Germany; ${ }^{7}$ Department of Neuropathology, Heidelberg University Hospital, 69120 Heidelberg, Germany;

${ }^{8}$ Department of Developmental Neurobiology, St. Jude Children's Research Hospital, Memphis, Tennessee, USA; ${ }^{9}$ Institut Curie, PSL Research University, UMR 3347, Centre National de la Recherche Scientifique (CNRS), U1021, Institut National de la Santé et de la Recherche Médicale (INSERM), Orsay 91405, France; ${ }^{10}$ Université Paris Sud, Université, UMR 3347, CNRS, U1021, INSERM, Orsay 91405, France; ${ }^{11}$ Princess Maxima Center for Pediatric Oncology, 3584 CS Utrecht, The Netherlands; ${ }^{12}$ Department for Paediatric Oncology and Haematology, Charité University Medicine, 13354 Berlin, Germany; ${ }^{13}$ Department for Pediatric Oncology and Hematology, Universitätsklinikum des Saarlandes, 66421 Homburg, Germany; ${ }^{14}$ Pediatric Haematology and Oncology, Pediatrics III, University Hospital of Essen, 45147 Essen, Germany; ${ }^{15}$ Department of Pediatric Hematology and Oncology, Heidelberg University Hospital, 69120 Heidelberg, Germany
\end{abstract}

Medulloblastoma is a malignant childhood brain tumor arising from the developing cerebellum. In Sonic Hedgehog (SHH) subgroup medulloblastoma, aberrant activation of SHH signaling causes increased proliferation of granule neuron progenitors (GNPs), and predisposes these cells to tumorigenesis. A second, cooperating genetic hit is often required to push these hyperplastic cells to malignancy and confer mutation-specific characteristics associated with oncogenic signaling. Somatic loss-of-function mutations of the transcriptional corepressor $B C O R$ are recurrent and enriched in SHH medulloblastoma. To investigate BCOR as a putative tumor suppressor, we used a genetically engineered mouse model to delete exons 9/10 of $B$ cor $\left(B \operatorname{cor}{ }^{\Delta E 9-10}\right)$ in GNPs during development. This mutation leads to reduced expression of C-terminally truncated BCOR $\left(\mathrm{BCOR}^{\Delta \mathrm{E} 9-10}\right)$. While Bcor $^{\Delta E 9-10}$ alone did not promote tumorigenesis or affect GNP differentiation, Bcor ${ }^{\triangle E 9-10}$ combined with loss of the SHH receptor gene Ptch1 resulted in fully penetrant medulloblastomas. In $\operatorname{Ptch1}^{+/-} ; B \operatorname{Bcor}{ }^{\Delta E 9-10}$ tumors, the growth factor gene Igf2 was aberrantly upregulated, and ectopic Igf2 overexpression was sufficient to drive tumorigenesis in Ptch1 ${ }^{+/-}$GNPs. BCOR directly regulates Igf2, likely through the PRC1.1 complex; the repressive histone mark H2AK119Ub is decreased at the Igf2 promoter in Ptch1 ${ }^{+/} ;$Bcor $^{\Delta E 9-10}$ tumors. Overall, our data suggests that BCOR-PRC1.1 disruption leads to Igf2 overexpression, which transforms preneoplastic cells to malignant tumors.

[Keywords: BCOR; brain tumor; cerebellar granule cells; PRC1.1 complex; medulloblastoma; mouse model]

Supplemental material is available for this article.

Received February 11, 2020; revised version accepted July 10, 2020.

${ }^{16}$ These authors contributed equally to this work. Corresponding authors: d.kawauchi@ncnp.go.jp; paul.northcott@stjude.org

Article published online ahead of print. Article and publication date are online at http://www.genesdev.org/cgi/doi/10.1101/gad.337584.120.
(C) 2020 Kutscher et al. This article is distributed exclusively by Cold Spring Harbor Laboratory Press for the first six months after the full-issue publication date (see http://genesdev.cshlp.org/site/misc/terms.xhtml). After six months, it is available under a Creative Commons License (Attribution-NonCommercial 4.0 International) as described at http://creativecommons.org/licenses/by-nc/4.0/. 
Pediatric cancer is the number one cause of disease-related death in children, and brain tumors are the most common pediatric solid tumor (Udaka and Packer 2018). Medulloblastoma is a malignant embryonal tumor of the cerebellum that is comprised of at least four biologically and clinically distinct molecular subgroups (Northcott et al. 2012). One subgroup, Sonic Hedgehog (SHH) medulloblastoma, arises through genetic alterations that activate the $\mathrm{SHH}$ signaling pathway, including mutations in the $\mathrm{SHH}$ receptor gene PTCH1, activating mutations in the transmembrane protein-coding gene Smoothened (SMO), mutations in the negative regulator gene SUFU, or amplification of the transcription factor genes GLI2 or MYCN (Hahn et al. 1996; Johnson et al. 1996; Goodrich et al. 1997; Kenney et al. 2003; Kool et al. 2014).

While constitutive activation of $\mathrm{SHH}$ signaling is required for $\mathrm{SHH}$ medulloblastoma formation, studies in mice have demonstrated that additional genetic hits are required for the malignant transformation of cerebellar granule neuron progenitors (GNPs) in Ptch1 heterozygous animals (Oliver et al. 2005; Kessler et al. 2009; TamayoOrrego et al. 2016; Tamayo-Orrego and Charron 2019). Depending on the nature of these mutations, the molecular and cellular properties of the tumor may change (Vogelstein and Kinzler 1993). More importantly, understanding how these cooperating mutations contribute to malignancy may reveal new therapeutic opportunities for affected patients.

We and others previously identified recurrent inactivating mutations targeting the transcriptional corepressor gene $B C O R$ in SHH medulloblastoma (Kool et al. 2014; Northcott et al. 2017; Waszak et al. 2020), but the mechanism(s) underlying $B C O R$-associated medulloblastoma formation remain unclear. $B C O R$ aberrations are implicated in a variety of pediatric cancers, including acute myeloid leukemia, retinoblastoma, sarcomas, glioblastomas, and CNS high-grade neuroepithelial tumor with $B C O R$ alteration (CNS-HGNET-BCOR) (Grossmann et al. 2011; Astolfi et al. 2015; Kooi et al. 2016; Sturm et al. 2016; Mackay et al. 2017; Santiago et al. 2017). Altogether, deregulation of $B C O R$ is implicated in at least 18 different tumor histotypes of pediatric cancer (Astolfi et al. 2019), demonstrating the urgency in understanding the role(s) of $B C O R$ in normal cellular processes and tumorigenesis.

BCOR was originally identified based on its interaction with the zinc finger transcription factor BCL6 and subsequently shown to be a component of a noncanonical Polycomb-repressive complex (PRC1.1) (Huynh et al. 2000; Gearhart et al. 2006; Cao et al. 2016). BCL6 can recruit BCOR to DNA via the BCL6-interacting motif found in the N-terminal half of BCOR (Fig. 1A; Ghetu et al. 2008). The $C$ terminus of BCOR contains nonankyrin repeats, ankyrin repeats, and the PUFD, which binds the Polycomb group protein PCGF1 (Fig. 1A; Junco et al. 2013). These regions and their associated interacting partners are conserved in BCOR in mice (Huynh et al. 2000; Wamstad and Bardwell 2007; Wamstad et al. 2008).

BCL6 has been implicated previously in cerebellar development in mice (Tiberi et al. 2014). Overexpression of human BCL6 suppresses medulloblastoma formation in vivo in mice, perhaps through interaction with BCOR at Gli1/Gli2 promoters (Tiberi et al. 2014), although recurrent $B C L 6$ mutations have not been identified in $\mathrm{SHH}$ medulloblastoma patient samples. While the interaction between BCOR and BCL6 may be one mode of BCORmediated tumor suppression, there are also truncating mutations in $B C O R$ in human tumors that occur after the BCL6 binding motif (Fig. 1A; Kool et al. 2014; Northcott et al. 2017; Waszak et al. 2020), suggesting that there may be other modes of BCOR-mediated tumor suppression. C-terminal truncation of the PUFD may lead to an inability to recruit PCGF1, which together with RING1B, ubiquitinates histone $\mathrm{H} 2 \mathrm{~A}$ at lysine 119 (H2AK119Ub) to repress transcription of target genes (Gearhart et al. 2006). Therefore, we set out to determine whether BCOR function within the PRC1.1 complex may be one mechanism of tumor suppression in $\mathrm{SHH}$ medulloblastoma.

Here we show that genetic ablation of exons 9 and 10 of $B \operatorname{cor}\left(B \operatorname{cor}^{\triangle E 9-10}\right)$ in GNPs does not disrupt granule neuron differentiation in mice. Instead, $B \operatorname{cor}^{\triangle E 9-10}$ cooperates with inactivation of Ptch1 to potentiate aggressive medulloblastoma formation. BCOR occupies the Igf2 promoter region in $P$ tch $1^{+/}$tumors, and chromatin occupancy is greatly reduced in $\operatorname{Ptch}^{+/-} ; B \operatorname{cor}^{\triangle E 9-10}$ tumors. This reduction is strongly associated with aberrant up-regulation of Igf2 in these tumors to drive tumorigenesis. Additionally, $\mathrm{BCOR}^{\triangle \mathrm{E} 9-10}$ no longer binds PCGF1 or RING1B, the catalytic subunit of the PRC1.1 complex, which leads to a global reduction of the repressive histone mark $\mathrm{H} 2 \mathrm{AK} 119 \mathrm{Ub}$, and a reduction of this mark specifically at the Igf2 promoter, compared with $P t c h 1^{+/-}$tumors. Our work demonstrates that loss of the $\mathrm{C}$ terminus of BCOR disrupts PRC1.1 complex function to drive $\mathrm{SHH}$ medulloblastoma formation in mice.

\section{Results}

BCOR is recurrently comutated with members of the SHH pathway in pediatric SHH medulloblastoma

We and others previously identified $B C O R$ as recurrently mutated or deleted in $8 \%$ of pediatric SHH medulloblastomas (Kool et al. 2014; Northcott et al. 2017; Waszak et al. 2020). We mapped the identified BCOR mutations from these published data sets to the BCOR coding sequence (Fig. 1A) and determined that the C-terminal PUFD may be absent due to the introduction of premature STOP codons in nine out of 13 cases (Table 1). In the majority of cases (10 out of 13), BCOR is comutated with members of the SHH pathway, including PTCH1 (5/10), GLI2 (one out of 10), SMO (two out of10), SUFU (two out of 10), or $\mathrm{SHH}$ gene amplification (one out of 10). BCOR is an $\mathrm{X}$-linked gene, and the majority of affected patients were male (nine out of 13). Given its potential as a cooperating mutation in SHH medulloblastoma formation, we investigated the functional relevance of $B C O R$ mutations during normal cerebellar development and tumorigenesis, specifically focusing on a potential role of the C-terminal domain in tumor suppression. 

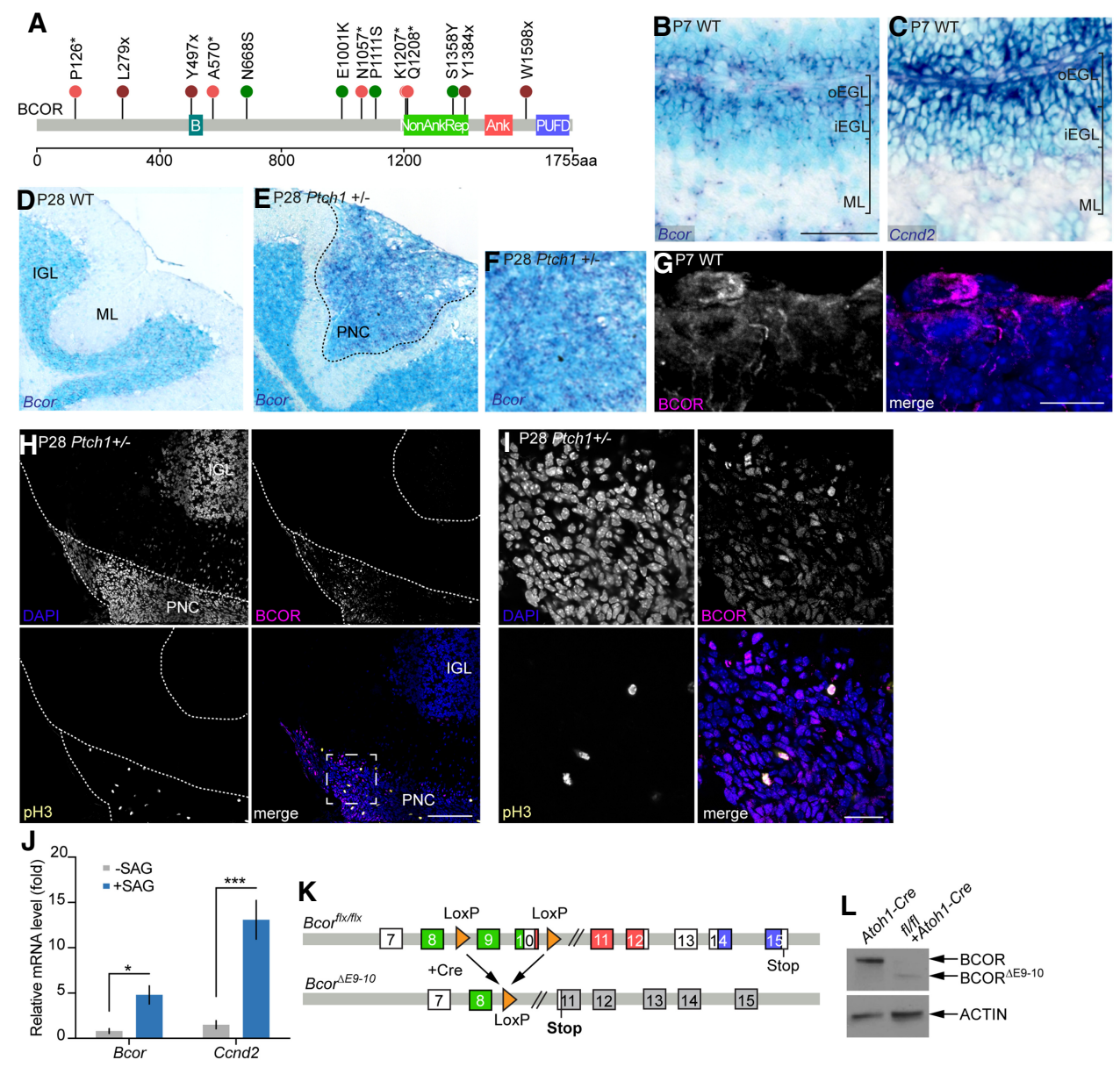

Figure 1. Conditional Bcor mouse model is an appropriate model for human BCOR mutations. $(A)$ BCOR mutations identified in pediatric medulloblastoma samples. (B) BCL6-binding domain; (NonAnkRep) nonankyrin repeats; (Ank) Ankyrin repeats; (PUFD) PCGF Ublike fold discriminator domain; (maroon lollipop) nonsense mutation; (pink lollipop) frame shift mutation; (green lollipop) point mutation. $(B, C)$ In situ hybridization (dark blue) of antisense $B$ cor probe $(B)$ and $C$ cnd2 probe $(C)$ at $\mathrm{P} 7 \mathrm{in}$ Bl6N wild-type $(\mathrm{WT})$ mice. Scale bar, $50 \mu \mathrm{m}$. (oEGL) Outer external granule layer; (iEGL) inner external granule layer; $(\mathrm{ML})$ molecular layer. Nuclei counterstained with methyl green. $(D, E)$ In situ hybridization of antisense Bcor probe at P28 in WT $(D)$ and in $P t c h 1^{+/-}(E)$ mice. Scale bar, $200 \mu \mathrm{m}$. (IGL) Inner granule layer; $(\mathrm{ML})$ molecular layer; (PNC) preneoplastic cells. Nuclei counterstained with methyl green. $(F)$ Increased magnification of preneoplastic region in $E$. Scale bar, $100 \mu \mathrm{m}$. (G, left) Immunohistochemistry using an anti-BCOR antibody (pink) at P7. (Right) DAPI stains nuclei in blue (merged). Maximum intensity projection of $16-\mu \mathrm{m}$-thick cerebellar section. Scale bar, $10 \mu \mathrm{m}$. $(H)$ Immunohistochemistry using an anti-BCOR antibody (pink) and an anti-pH3 antibody (yellow) at P28 in the preneoplastic lesion of Ptch1 $1^{+/}$animals. DAPI stains nuclei in blue. Single z-slice. Scale bar, $100 \mu \mathrm{m}$. (PNC) Preneoplastic cells; (IGL) inner granule layer. (I) Increased magnification of boxed region in $H$. Scale bar, $20 \mu \mathrm{m}$. (J) Relative mRNA expression of Bcor and Ccnd2 (quantitative PCR) of P7 granule neuron progenitors cultured in the absence (gray) or presence (blue) of Smoothened agonist (SAG). $\left.\left(^{*}\right) P<0.05 ;{ }^{* * *}\right) P<0.001$, Student's $t$-test. Bars represent mean \pm SEM. $N=$ 6. $(K)$ Location of LoxP sites in Bcor. Expression of Cre removes exons 9 and 10, and leads to an early translation stop. Color scheme for $B$ cor domains same as in $A$. $(L)$ anti-BCOR Western blot of purified granule neuron progenitors in wild-type (Atoh1-Cre only) cells and in $B c r^{\text {floxed/floxed }}+$ Atoh1-Cre $\left(B \operatorname{cor}^{\Delta E 9-10}\right)$ cells. Anti-ACTIN was used as the loading control. See also Supplemental Figures S1 and S2.

\section{Bcor is expressed in proliferating murine GNPs}

We first determined whether Bcor is normally expressed in developing cerebellar GNPs in mice, the cell of origin for SHH medulloblastoma (Schüller et al. 2008; Yang et al. 2008). Using RNA in situ hybridization (ISH), we found that Bcor is expressed at low levels in the outer external granule layer of the $\mathrm{P} 7$ cerebellum (Fig. 1B), in a similar domain as actively cycling cells (Ccnd2, Fig. 1C). After cerebellar development is complete, however, $B c o r$ is no longer expressed (P28, Fig. 1D).
In mice heterozygous for the $\mathrm{SHH}$ receptor gene Ptch1 (written as $P t c h 1^{+1-}$ here), preneoplastic lesions form in $85 \%$ of mice by $\mathrm{P} 21$, but the majority regress by $\mathrm{P} 42$ (Corcoran et al. 2008; Kessler et al. 2009). We found that Bcor is expressed in these preneoplastic lesions but not in adjacent normal tissue (Fig. 1E,F).

To determine whether BCOR protein is expressed in a similar manner, we performed immunohistochemistry using an anti-BCOR antibody. At P7, BCOR is detected in high levels in dividing GNPs (one to two cells per 
Table 1. BCOR mutations in SHH medulloblastoma patient samples

\begin{tabular}{|c|c|c|c|c|c|c|c|}
\hline BCOR mutation & Co-occurring alterations & Sex & Age (years) & Affymetrix & RNA-seq & Histology & Reference \\
\hline $\mathrm{P} 126^{\mathrm{a}}$ & SMO & M & 2 & No & Yes & Unknown & Waszak et al. 2020 \\
\hline L279delX & $S H H a m p^{b}$ & $\mathrm{~F}$ & 2 & No & No & Desmoplastic & Kool et al. 2014 \\
\hline Y497delX & SMO & M & 3 & No & Yes & Desmoplastic & Northcott et al. 2017 \\
\hline $\mathrm{A} 570^{\mathrm{a}}$ & None ID'd & M & 1 & Yes & No & Unknown & Northcott et al. 2017 \\
\hline N668S & PTCH1 $^{\mathrm{b}}$ & $\mathrm{F}$ & 43 & No & No & Classic & Kool et al. 2014 \\
\hline E1001K & SUFU, DDX $3 X, P T E N$ & M & 5 & No & No & Unknown & Waszak et al. 2020 \\
\hline $\mathrm{N} 1057^{\mathrm{a}}$ & PTCH1, DDX $3 X$ & M & 16 & No & No & Unknown & Northcott et al. 2017 \\
\hline P1111S & None ID'd $\mathrm{d}^{\mathrm{b}}$ & $\mathrm{F}$ & 2 & Yes & No & Desmoplastic & Kool et al. 2014 \\
\hline $\mathrm{K} 1207^{\mathrm{a}}$ & $D D X 3 X$ & M & 7 & No & No & Classic & Northcott et al. 2017 \\
\hline Q1208 ${ }^{\mathrm{a}}$ & SUFU, ZIC2 & M & 1 & No & No & Unknown & Northcott et al. 2017 \\
\hline S1358Y & PTCH1, SMARCB1 & $\mathrm{F}$ & 27 & Yes & Yes & Unknown & Northcott et al. 2017 \\
\hline Y1384X & PTCH1 & M & 4 & No & Yes & Unknown & Waszak et al. 2020 \\
\hline W1598X & PTCH1,GLI2, PTEN, DDX3X & M & 25 & No & No & Desmoplastic & Northcott et al. 2017 \\
\hline
\end{tabular}

(delX) Deletion resulting in nonsense mutation.

${ }^{a}$ Frameshift mutation. (X) Nonsense mutation; (M) male; (F) female; (None ID'd) none identified.

${ }^{b}$ Panel sequencing. (amp) Amplification; (unknown) histology type unknown/not available.

$10^{4}-\mu \mathrm{m}^{2}$ area), and at lower levels throughout the outer EGL (average number of $\mathrm{BCOR}^{+}$cells in the EGL $36 \% \pm$ $19 \%, N=12$ regions) (Fig. 1G; Supplemental Fig. S1A,B). At P28, we did not detect BCOR at appreciable levels in wild-type animals (Supplemental Fig. S1C), but we did detect BCOR protein in $\mathrm{Ptch}^{+/-}$preneoplastic lesions (Fig. 1H,I), with highest expression in mitotic cells (phospho-histone $\mathrm{H} 3$ [pH3]-positive). In some preneoplastic lesions, BCOR protein was detected in up to $100 \%$ of cells (average number of $\mathrm{BCOR}^{+}$cells $62 \% \pm 25 \%, N=12$ regions).

To determine the effect of proliferation on Bcor expression, we examined Bcor RNA levels in GNPs cultured with or without a Smoothened agonist (SAG) in vitro. Bcor expression is $\sim 6 \times$ higher in proliferating GNPs (with SAG) compared with differentiated cells (without SAG) (Fig. 1J, Ccnd2 as positive control). Taken together, the expression pattern demonstrates that Bcor is up-regulated in actively proliferating cells.

Mouse NIH/3T3 cells are sensitive to SHH signaling, which allows us to determine whether Bcor is regulated by SHH signaling, either directly or indirectly. We treated $\mathrm{NIH} / 3 \mathrm{~T} 3$ cells with SAG or DMSO control and found that while Gli1 is significantly up-regulated after SAG treatment, Bcor levels remain unchanged (Supplemental Fig. S1D). These results suggest that Gli1 and SHH signaling do not directly (or indirectly) regulate Bcor, and that instead Bcor expression seems to be associated with cell cycle states.

To further examine the likelihood of SHH-independent expression of $B c o r$, we examined mouse GLI1/GLI2 target genes using ChIP-Atlas (http://chip-atlas.org), a Web tool that predicts protein-specific gene regulation, based on the binding profiles of public chromatin immunoprecipitation (ChIP) sequencing data for a particular gene locus (Oki et al. 2018). Using this program, we did not detect GLI1 or GLI2 binding within $\pm 10 \mathrm{~kb}$ of the transcriptional start site of $B c o r$. We also examined more closely the data from Lee et al. (2010), where GLI1 binding sites were mapped by ChIP-seq in wild-type GNPs and in Ptch1-driven medulloblastoma. They also did not detect Bcor as a GLI1 target gene, supporting the idea that $B c o r$ activation is independent of GLI-mediated SHH signaling pathway.

\section{BCL6-related cerebellar functions are maintained in the Bcor ${ }^{\Delta E 9-10}$ mouse model}

BCOR and its N-terminal interaction with BCL6 has been previously implicated in SHH medulloblastoma in mice (Tiberi et al. 2014). Using our mouse model, we sought to determine whether any BCL6-related functions of BCOR were disrupted, as this would allow us to distinguish the role of the C-terminal domain in BCOR-mediated tumor suppression, outside of BCL6-mediated processes. We were especially interested in this given the majority of $B C O R$ mutations in SHH medulloblasto$\mathrm{ma}$ are predicted to disrupt C-terminal function (Fig. 1A). To investigate the functional relevance of a C-terminal deletion of BCOR during GNP development and tumorigenesis, we used a genetically engineered mouse strain to generate Cre inducible excision of $B c o r$ exons 9 and 10 in Atoh1-positive GNPs $\left(B c o r^{\triangle E 9-10}\right)$ (Fig. $1 \mathrm{~K} ; \mathrm{Ham}$ line et al. 2020). At the protein level, this excision results in reduced expression of a truncated BCOR protein (Fig. $1 \mathrm{~L})$.

We investigated whether BCL6-related functions were disrupted in our mouse model. We attempted to coimmunoprecipitate (co-IP) BCL6 using an anti-BCOR antibody in purified P7 GNPs, but we did not detect BCL6 by Western blot in either the input or the IP lanes. Therefore, we used a heterologous system to investigate whether $\mathrm{BCOR}^{\triangle \mathrm{E} 9-10}$ interacts with BCL6. We overexpressed HAtagged full-length mouse BCOR or HA-tagged mouse $\mathrm{BCOR}^{\triangle \mathrm{E} 9-10}$ with mouse BCL6 in HEK293T cells and performed co-IP using bulk protein extract. We found that $\mathrm{BCOR}^{\triangle \mathrm{E} 9-10}$ interacts with BCL6 in vitro (Supplemental Fig. S2A), suggesting that the BCL6 interacting motif of 
$\mathrm{BCOR}^{\triangle \mathrm{E} 9-10}$ may still be functional if these proteins are coexpressed in the same cells.

Next, we examined whether any known BCL6-related developmental functions are affected in vivo in our Bcor $^{\triangle E 9-10}$ mouse model. Bcl6 is required for timely GNP differentiation (Tiberi et al. 2014), so we first tested whether differentiation is affected in $B \operatorname{cor}^{\triangle E 9-10}$ GNPs. In contrast to Bcl6 knockout animals (Tiberi et al. 2014), we found that Bcor ${ }^{\Delta E 9-10}$ mice do not exhibit defects in GNP differentiation or migration (Supplemental Fig. S2), further suggesting that previously reported BCL6-related functions in the cerebellum are largely unaffected in our mouse model. We found no differences between wildtype and $B \operatorname{cor}^{\triangle E 9-10}$ animals in the total number of GNP cells at P7 (PAX6-positive, Supplemental Fig. S2B-D) or proliferating cells (Ki67-positive) (Supplemental Fig. S2E-G). We found comparable numbers of $\mathrm{NeuN}^{+}$cells between wild-type and $B \operatorname{cor}^{\Delta E 9-10}$ cerebella at P7 (Supplemental Fig. S2H-J). We also found no differences in proliferation or cell cycle exit $2 \mathrm{~h}$ after a single dose of EdU at P7 (Supplemental Fig. S2K-P). We examined migration of granule neurons at P28 following a single EdU pulse at P7 and found no differences between wild-type and Bcor $^{\Delta{ }^{E-10}}$ animals (Supplemental Fig. S2Q-U).

To further confirm that BCL6-dependent roles of BCOR are largely unaffected in $B c o r^{\triangle E 9-10}$ GNPs, we examined expression of known BCL6/BCOR-regulated genes (Tiberi et al. 2014). Loss of Bcl6 in cerebellar cells resulted in an increase in Gli1 and Gli2 expression, and both BCOR and BCL6 were bound at these promoter regions (Tiberi et al. 2014). To determine whether BCOR ${ }^{\triangle \mathrm{E} 9-10}$ affects expression of these and other SHH-pathway related genes in GNPs, we isolated total RNA from wild-type and Bcor $^{\triangle E 9-10}$ GNPs and evaluated target gene expression using qPCR. In contrast to Bcl6 loss (Tiberi et al. 2014), loss of the region encoding the C-terminal domain of BCOR did not activate expression of Gli1, Gli2, Ccnd1, Ptch1, or Mycn (Supplemental Fig. S2V), suggesting that $\mathrm{BCOR}^{\triangle \mathrm{E} 9-10}$ does not influence BCL6-related SHH signaling repression in GNPs. Taken together, our Bcor ${ }^{\triangle E 9-10}$ mouse model allows us to assess the role of BCOR in GNPs, and in particular the involvement of the C terminus and PRC1.1 in medulloblastoma formation.

Bcor $^{\Delta \mathrm{E} 9-10}$ significantly reduces latency and increases penetrance of Ptch1-associated medulloblastoma

Next, we examined whether Bcor ${ }^{\Delta E 9-10}$ affects tumor formation in conjunction with Ptch1 inactivation. Deletion of the region encoding the C-terminal domain of BCOR in GNPs by itself did not promote medulloblastoma (Fig. 2A). Similar to previous studies (Goodrich et al. 1997), heterozygous mutations in Ptch1 resulted in spontaneous medulloblastoma formation in $35 \%$ of animals, with a median latency of $179 \mathrm{~d}$ (Fig. 2A). In these mice, the second copy of Ptch1 is inactivated, with additional secondary mutations in other genes (Tamayo-Orrego et al. 2016). Combining Bcor ${ }^{\Delta E 9-10}$ with Ptch1 mutations resulted in tumorigenesis with $100 \%$ penetrance and a median latency of $75 \mathrm{~d}(P<0.0001$, log-rank [Mantel-
Cox] test) (Fig. 2A). There was a histological switch from classic in Ptch $1^{+/-}$tumors $(N=3 / 3)$ (Fig. $\left.2 \mathrm{~B}\right)$ to the more aggressive histological subtype, large cell anaplastic (LCA), in Ptch $^{+/-} ;$Bcor $^{\Delta E 9-10}$ tumors $(N=3 / 3)$ (Fig. 2C), although $B C O R$ mutated human tumors typically exhibit classic or desmoplastic histology (Table 1). To confirm that $P t c h 1^{+/-} ; B c o r^{\Delta E 9-10}$ mouse tumor cells had higher tumorigenic potential, we transplanted $8 \times 10^{5}$ cells from either Ptch $1^{+/-}$tumors or Ptch1 ${ }^{+/-} ; B \operatorname{cor}{ }^{\Delta E 9-10}$ tumors into the cerebella of immunodeficient mice. $P t c h 1^{+/-}$; $B$ cor $^{\triangle E 9-10}$ tumor cells reestablished tumors considerably faster than $P t c h 1^{+/-}$tumor cells (median latency $26 \mathrm{~d}$ vs. $150 \mathrm{~d}, P<0.0001$, log-rank [Mantel-Cox] test) (Fig. 2D).

To determine whether Bcor ${ }^{\Delta E 9-10}$ drives early transformation events, we next examined the number of preneoplastic lesions in the cerebella of P28 mice. Ptch1 $1^{+-}$ animals carry a lac $Z$ gene that disrupts one copy of the Ptch1 locus, and preneoplastic cell clusters, where the $P t c h 1$ promoter is active, can be identified by $\beta$-galactosidase staining (Fig. 2E-H; Goodrich et al. 1997; Oliver et al.

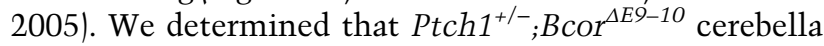
have twice the number of the lesions compared with Ptch $1^{+/-}$cerebella alone $(N=3$ cerebella per genotype, $P=0.0232$, Student's $t$-test) (Fig. 2I). This result suggests that a greater proportion of GNPs continue to proliferate in the absence of full-length Bcor and Ptch1 compared with Ptch1 loss alone, increasing the likelihood of malignant transformation. Intriguingly, loss of Ptch1 together with $B \operatorname{cor}^{\triangle E 9-10}$ resulted in a decrease of SHH-related gene expression compared with Ptch1 alone (P7 GNPs, Supplemental Fig. S2V), suggesting deregulation of alternative pathway(s) may be required to promote malignant transformation.

\section{Igf2 is up-regulated in murine medulloblastomas}

Based on our qPCR data (Supplemental Fig. S2V), SHH pathway genes are likely not the only drivers in Ptch $1^{+/-} ; B \operatorname{cor}^{\Delta E 9-10}$ tumors. To identify alternative signaling pathways or genes that may contribute to tumorigenesis in this context, we used RNA sequencing to identify differentially expressed genes in $\mathrm{Ptch}^{+/-} ; \mathrm{Bcor}^{\Delta E 9-10}$ tumors compared with Ptch $1^{+/-}$tumors. Unsupervised clustering of transcriptome profiles demonstrated a clear difference between the tumor models (Supplemental Fig. S3A) and differential expression analysis revealed possible drivers (Supplemental Table S1).

We performed a gene ontology analysis on the differentially expressed genes using DAVID, focusing on biological processes and the KEGG pathway (Supplemental Table S2). We observed enrichment of various epigenetic activities, such as transcription regulation, and enrichment of cell proliferation pathways in the $\mathrm{Ptch}^{+/-}$; Bcor $^{\Delta E 9-10}$ tumors (Supplemental Table S2). The SHH pathway was not preferentially enriched, since likely both models depend on $\mathrm{SHH}$ signaling for tumorigenesis. We also found known cancer signaling pathways up-regulated in $\mathrm{Ptch}^{+/-} ; \mathrm{BcOr}^{\Delta E 9-10}$ tumors, including the HIPPO signaling pathway, MAPK pathway, and PI3K-Akt signaling pathway members (Supplemental Table S2). 

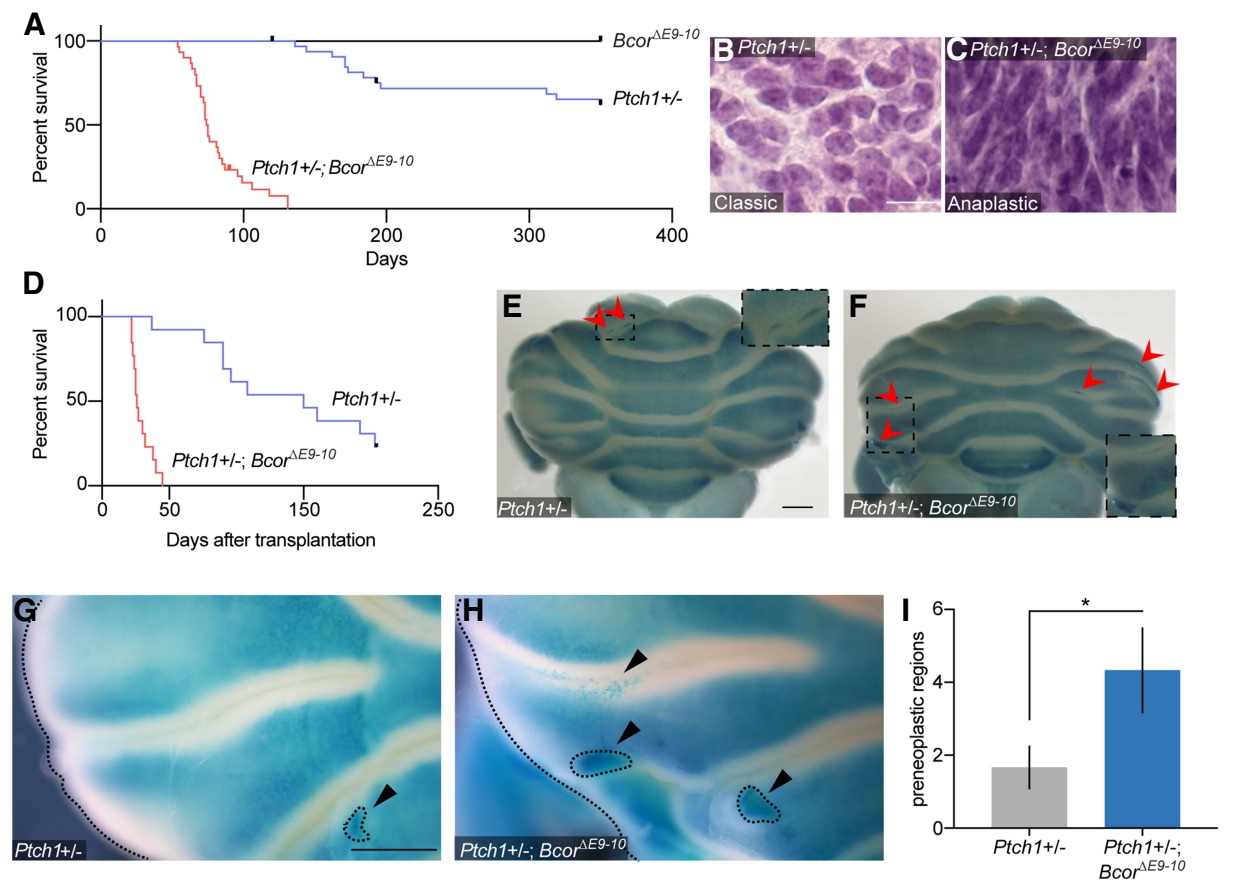

Figure 2. $B c o r^{\Delta E 9-10}$ significantly reduces latency and increases penetrance in Ptch1-driven medulloblastoma. $(A)$ Survival curve of

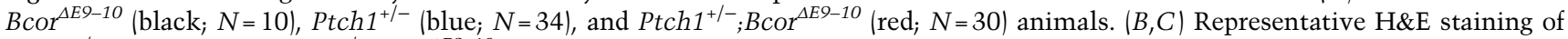
$P t c h 1^{+/-}$tumor $(B)$ and $P t c h 1^{+/-} ; B c o r^{\Delta E 9-10}$ tumor $(C)$. Notice typical round cells of classic histology $(B)$ versus larger, pleomorphic nuclei of anaplastic histology $(C)$. Scale bar, $10 \mu \mathrm{m}$. (D) Survival curve of immunodeficient animals transplanted with $8 \times 10^{5}$ cells from $P t c h 1^{+/-}$ (blue; $N=13)$ and $P t c h 1^{+-} ; B c o r^{\Delta E 9-10}($ red; $N=13)$ tumors. $(E, F)$ Representative examples of $\beta$-galactosidase staining (lacZ present in Ptch1 locus) of P28 Ptch1 ${ }^{+/-}$cerebellum $(E)$ and $P t c h 1^{+/-} ; B \operatorname{cor}^{\Delta E 9-10}$ cerebellum $(F)$. (Red arrows) Preneoplastic lesions. (Inset) Magnified view of preneoplastic lesion. Scale bar, $1000 \mu \mathrm{m}$. $(G, H)$ Increased magnification of $\beta$-galactosidase staining similar to $E$ and $F$. Preneoplastic lesions are noted by arrowheads and dotted lines. Scale bar, $500 \mu \mathrm{m}$. (I) Quantification of the number of preneoplastic lesions in $E$ and $F$. $\left(^{*}\right) P<0.05$, Student's $t$-test. Bars represent mean \pm standard deviation. $N=3$ cerebella per genotype.

Upon closer examination of the specific up-regulated genes, we focused on the growth factor Igf2 as a potential tumorigenic driver in these tumors. Igf2 was up-regulated $\sim 20$-fold in Ptch $^{+/-} ; B \operatorname{cor}^{\Delta E 9-10}$ tumors compared with Ptch $1^{+/-}$tumors $\left(\log _{2} \mathrm{FC}: \sim 4.4, P\right.$-adj $=5.26 \times 10^{-30}$ ) (Fig. $3 \mathrm{~A})$. We also verified Igf2 overexpression using Affymetrix microarray data from an additional cohort of samples (Supplemental Fig. S3B,C; Supplemental Table S1) and by performing qPCR on individual tumor samples (Fig. 3B). We found that IGF2 protein is expressed in $P t c h 1^{+/-}$; Bcor ${ }^{\Delta E 9-10}$ tumors, but not in P7 GNPs or Ptch1 ${ }^{+/-}$tumors (Fig. 3C).

Consistent with previous results (Corcoran et al. 2008), we did not detect Igf2 expression in P7 GNPs in wild-type animals (Fig. 3B). There was also no aberrant expression of Igf2 in P7 GNPs from either Ptch1 ${ }^{+/-}$or Ptch $1^{1+-} ;$ Bcor $^{\Delta E 9-}$ ${ }^{10}$ mice by qPCR (Fig. 3B) or ISH (Fig. 3D,E). Additionally, we did not detect Igf2 expression in $P t c h 1^{+/-}$preneoplastic lesions or in $\mathrm{Ptch}^{+/-}$tumors (Fig. 3B,F,H; Supplemental Fig. S3D). Instead, aberrant Igf2 expression was observed only in preneoplastic lesions of $\mathrm{Ptch1}^{+/-}$; $B c r^{\Delta E 9-10}$ animals (Fig. 3G) and in Ptch1 ${ }^{+/-} ; B \operatorname{Bcor}^{\Delta E 9-10}$ tumors (Fig. 3B,I; Supplemental Fig. S3E), suggesting that inactivation of both Ptch1 and Bcor are required for Igf2 upregulation.
IGF2 up-regulation is associated with BCOR mutations across multiple tumor types

To determine whether Igf2 up-regulation is a conserved mechanism across species, we examined human medulloblastoma samples in which both genomic and transcriptomic data are available (Table 1; Kool et al. 2014; Northcott et al. 2017; Waszak et al. 2020). To investigate the transcriptional similarities between our mouse model and the ICGC medulloblastoma human tumor cohort (Northcott et al. 2017), we compared gene expression profiles between mouse and human tumors. Unsupervised clustering showed that our mouse models correspond to the SHH medulloblastoma subgroup, as expected (Supplemental Fig. S4A). We next focused only on the ICGC SHH medulloblastoma tumors and restricted our comparisons with the differentially expressed genes between $\mathrm{Ptch}^{+/-}$ and $P$ tch1 ${ }^{+/-} ; B \operatorname{cor}^{49-10}$ mouse models. We observed that Ptch ${ }^{+/-} ; B \operatorname{cor}^{\Delta 9-10}$ mouse tumors clustered near ICGC sample MB12 (Supplemental Fig. S4B). In this sample, there was a somatic mutation in $B C O R$ (Y497delX) and a strong activation of IGF2 (Fig. 3J).

The $B C O R$ mutation in MB12 was confirmed using RNA-seq alignment data, and $B C O R$ was highly expressed in this sample compared with other $\mathrm{SHH}$ 

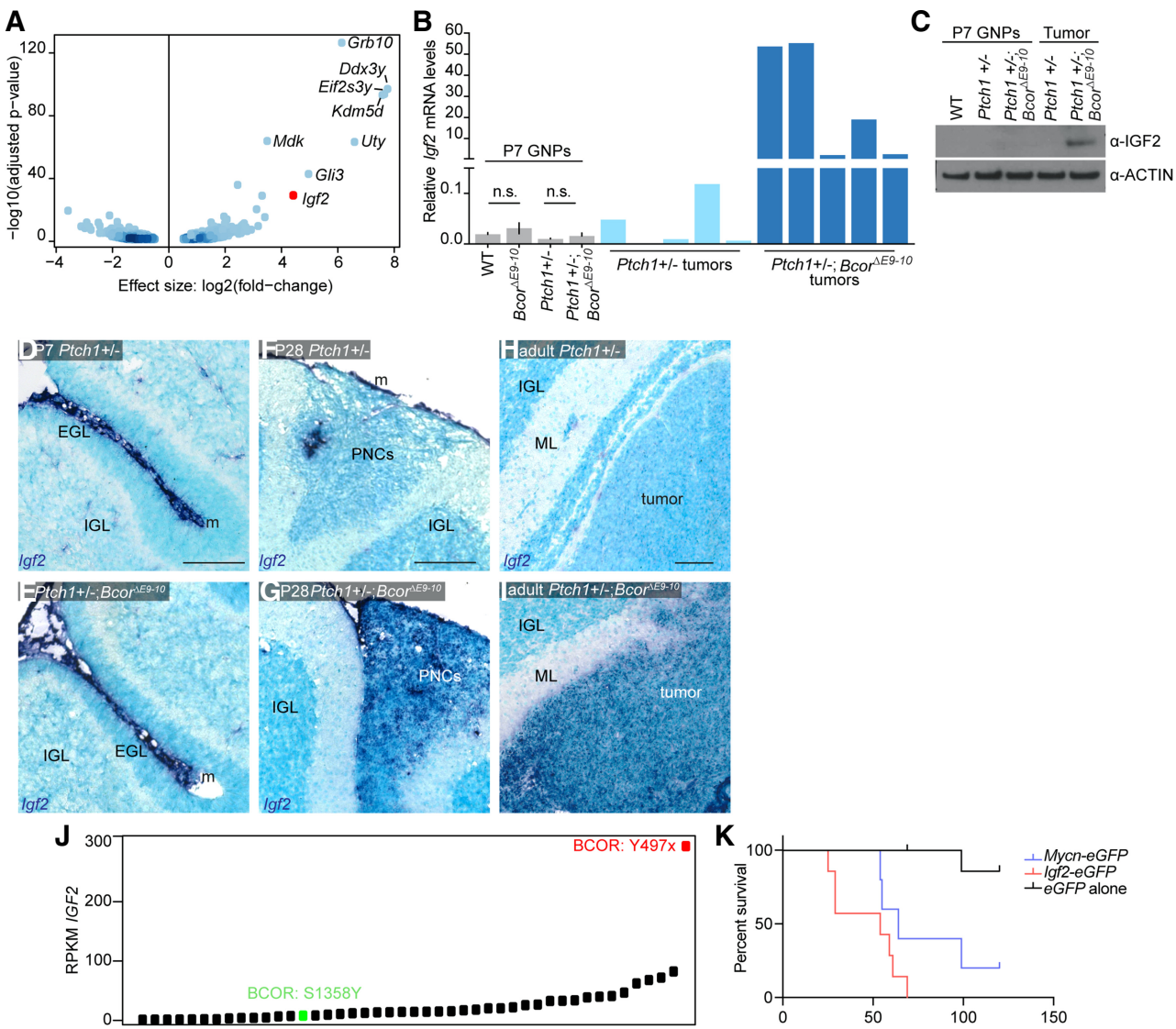

ICGC Medulloblastoma Cohort
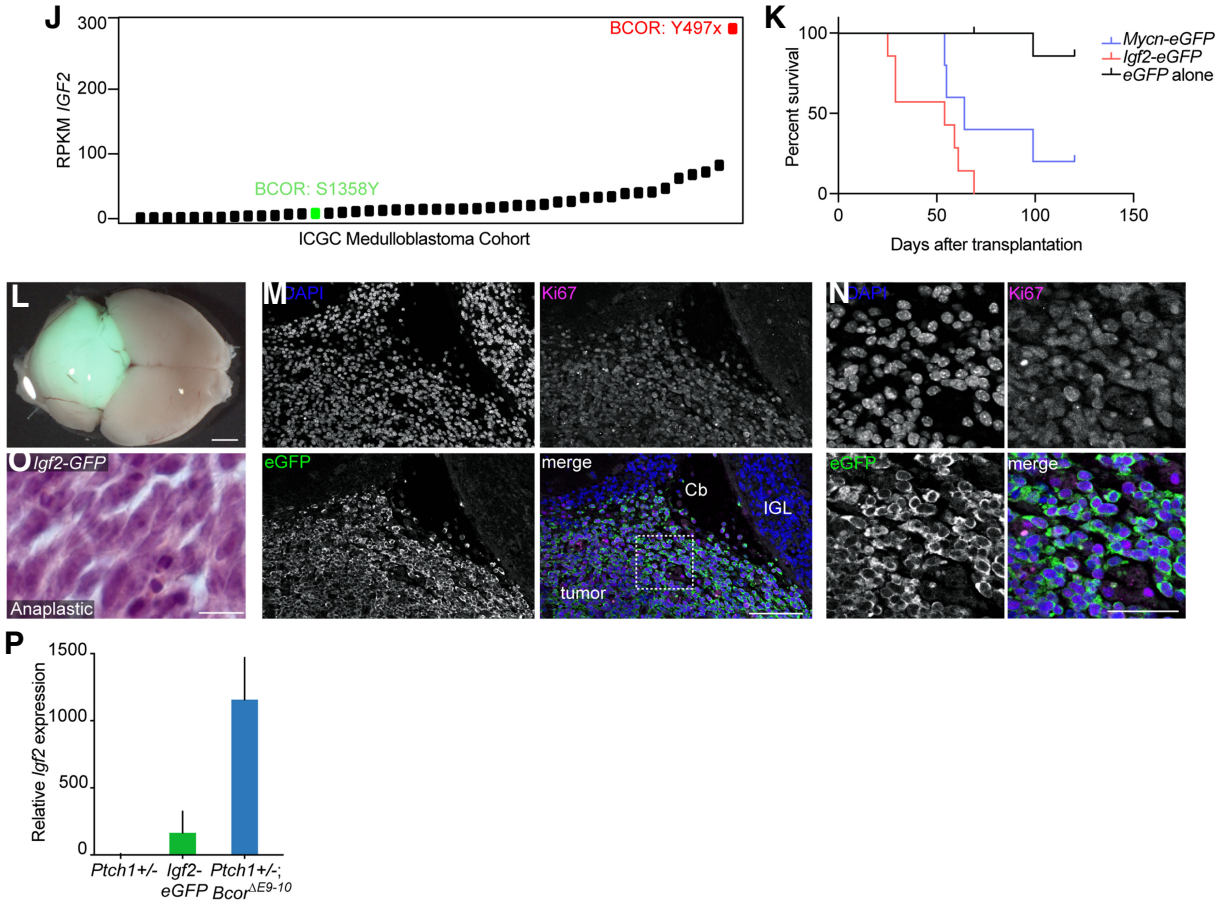

Figure 3. Igf2 is overexpressed in $P t c h 1^{+/-} ; B \operatorname{cor}{ }^{\Delta E 9-10}$ tumors. $(A)$ Volcano plot of differentially expressed genes between $P t c h 1^{+/-}$and Ptch $1^{+/} ;$;Bcor ${ }^{\Delta E 9-10}$ tumors. (B) Relative mRNA expression of Igf2 (qPCR) of P7 GNPs (gray) in the indicated genotypes, Ptch1 ${ }^{+/-}$tumors (light blue), and $P t c h 1^{+/-} ; B c o r^{\Delta E 9-10}$ tumors (dark blue). (n.s.) Not significant, Student's $t$-test. Bars of P7 GNPs represent mean \pm SEM. $N=$ 6 (WT, Bcor ${ }^{\Delta E 9-10}$ GNPs) or $N=8\left(\right.$ Ptch $^{+/-}{ }^{-}$, Ptch $\left.1^{+/-} ; B \operatorname{cor}^{\Delta E 9-10} \mathrm{GNPs}\right) .(C)$ Anti-IGF2 Western blot of total protein extracts from P7 GNPs or tumor samples from the indicated genotypes. Anti-ACTIN was used as the loading control. (D-I) ISH of antisense Igf2 probe (dark blue) in cerebella of animals from the indicated genotypes. (IGL) Inner granule layer; (EGL) external granule layer; (ML) molecular layer; (m) meninges; (PNC) preneoplastic cells. $(D, E)$ P7. Scale bar, $100 \mu \mathrm{m} .(F, G)$ P28. Scale bar, $200 \mu \mathrm{m} .(H, I)$ Adult tumor. Scale bar, $100 \mu \mathrm{m}$. $(J)$ Reads per kilobase of transcript per million mapped reads (RPKM) of IGF2 from the ICGC cohort of SHH medulloblastoma samples (Northcott et al. 2017). Black samples are wild type at the BCOR locus. (K) Survival curve of immunodeficient animals transplanted with $1 \times 10^{6} \mathrm{Ptch}^{+/-}$GNPs transduced with retrovirus overexpressing $e G F P$ alone (black; $\left.N=8\right)$, Igf2-eGFP (red; $\left.N=7\right)$, or Mycn-eGFP (blue; $N=5$ ). (L) Representative example of resulting Igf2-eGFP tumor from K. Scale bar, $2000 \mu \mathrm{m}$. (M) DAPI (top left; blue), eGFP (bottom left; green), and Ki67 (top right; magenta) immunohistochemistry of Igf2-eGFP-driven tumor. (IGL) Inner granule layer; (Cb) cerebellum. Scale bar, $100 \mu \mathrm{m}$. (N) Increased magnification of boxed region in $M$. Scale bar, $50 \mu \mathrm{m}$. (O) Representative histology sample of Igf2-eGFPdriven tumor. Scale bar, $10 \mu \mathrm{m}$. $(P)$ Relative mRNA expression of Igf2 in tumor cells from the indicated genotypes, Bars represent mean + SEM. $N=7$ for $P t c h 1^{+/}, N=4$ for Igf2-eGFP, or $N=5$ for Ptch1 ${ }^{+/-} ; B \operatorname{Bcor}{ }^{\Delta E 9-10}$. See also Supplemental Figures S3 and S4 and Supplemental Tables S1 and S2. 
medulloblastoma samples (Supplemental Fig. S4C). This result suggests that the C-terminal domain of BCOR may indeed be affected, as a previously characterized $B$ cor gene trap allele that lacks the $\mathrm{C}$ terminus is similarly up-regulated (Wamstad et al. 2008). Using our Affymetrix data set, we also observed that the P1111S mutation was coincident with high IGF2 overexpression, and A570* had moderate IGF2 expression (Supplemental Fig. S4D).

While we also had one sample in the ICGC cohort with a $B C O R$ missense mutation (S1358Y) that did not exhibit up-regulation of IGF2 (Fig. 3J; Supplemental Fig. S4D), this mutation was located within the preankyrin repeats and may not negatively affect BCOR C-terminal function (Fig. 1A). BCOR expression in this tumor was also reduced compared with the rest of the cohort (Supplemental Fig. $\mathrm{S} 4 \mathrm{C}$ ), and the mutation was detected in only $2 \%$ of the RNA-seq reads.

While both the ICGC and Affymetrix cohorts contained $B C O R$ mutated samples with high IGF2 expression, we also examined three additional $B C O R$ mutated samples from a recently published study (Waszak et al. 2020). For these three tumor samples, two had matching RNA-seq data (P126X and Y1384X). While we detected only the mutated reads for P126X in the RNA-seq data, wild-type $B C O R$ transcript was detected in $14 \%$ of the reads in the Y1384X tumor sample. Nevertheless, neither of the tumors exhibited up-regulation of IGF2, suggesting that there are additional mechanisms of $B C O R$-associated tumorigenesis independent of IGF2.

Because the $B C O R$ mutated samples only partially correlated with up-regulation of IGF2 (three out of six samples), we examined other tumor subtypes with $B C O R$ alterations. We focused on CNS-HGNET-BCOR tumors and clear cell sarcomas of the kidney (CCSK) tumors, as both of these tumor types have consistent alterations in the C-terminal domain of BCOR (internal tandem duplications [ITDs] in the PUFD [Paret et al. 2016; Wong et al. 2018]). Using a small cohort of CNS-HGNETBCOR samples analyzed as part of our ongoing INFORM study (Worst et al. 2016), we confirmed that these tumors have high levels of IGF2, (Supplemental Fig. S4E), similar to published results (Vewinger et al. 2019). In three out of three CNS-HGNET-BCOR tumor samples, we detect upregulation of BCOR itself (Supplemental Fig. S4F) and upregulation of $\mathrm{SHH}$ pathway-related genes (Supplemental Fig. S4G,H), similar to published results (Paret et al. 2016).

In the five CCSK samples, we also detected high levels of IGF2 (five out of five) (Supplemental Fig. S4E), up-regulation of BCOR (Supplemental Fig. S4F), and up-regulation of PTCH1 and GLI2 (Supplemental Fig. S4G,H), suggesting a role of $\mathrm{SHH}$ signaling in these tumors. In both cases, we compared expression of the target genes with group 3/4 medulloblastomas and $\mathrm{SHH}$ subgroup of medulloblasto$\mathrm{ma}$, all samples being wild type at the $B C O R$ locus.

\section{Igf2 overexpression is sufficient to drive tumorigenesis in $\mathrm{Ptch}^{+/-}$GNPS}

To verify that Igf2 overexpression is an oncogenic cofactor in $\mathrm{SHH}$ medulloblastoma formation /Corcoran et al.
2008), we transduced $P t c h 1^{+/-}$GNPs ex vivo with retroviruses encoding Igf2-IRES-eGFP, Mycn-IRES-eGFP as a positive control, or eGFP alone as a negative control. We transplanted $1 \times 10^{6}$ transduced cells of each condition into the cerebella of adult immunocompromised mice. Igf2-transduced cells formed aggressive tumors that developed significantly faster than cells transduced with $e G F P$ alone $(100 \%$ penetrance; median survival $=54 \mathrm{~d}$ for $\operatorname{Ig} f 2$, $P<0.0001$, log-rank [Mantel-Cox] test) (Fig. 3K). Proliferating tumor cells (Ki67-positive) were eGFP-positive (Fig. 3L-N). Histologically, these tumors resembled Ptch $1^{+/-}$; $B$ cor $^{\triangle E 9-10}$ tumors and all displayed an anaplastic histology (Fig. 3, cf. O $[N=3]$ and $\mathrm{B}, \mathrm{C}$ ).

Because Igf2 overexpression accelerated tumorigenesis in this model, we examined the level of Igf2 necessary for tumor induction and compared it with the expression level in the genetically engineered mouse models. We observed lower levels of Igf2 expression in the Igf2-IRESeGFP induced tumors compared with $P t c h 1^{+/-} ; B \operatorname{cor}^{\Delta E 9-10}$ genetic tumors (Fig. 3P). While this difference in Igf2 levels may be affected by the starting material used or mouse strain background, it also suggests the level of Igf2 in Ptch $^{+/-} ; B$ cor $^{\Delta E 9-10}$ tumors is more than sufficient to drive tumorigenesis.

$B C O R^{\Delta E 9-10}$ fails to interact with members of the PRC1.1 complex, and the PUFD of BCOR is required for Igf2

repression

The C-terminal domain of BCOR mediates its interaction with the PRC1.1 complex, a multi-protein complex that transfers ubiquitin to H2AK119 via the E3 ubiquitin ligase RING1B to repress target gene transcription (Wang et al. 2004, 2018). We tested whether the PRC1.1 complex is affected in $P$ tch $1^{+/-} ; B \operatorname{cor}^{\triangle E 9-10}$ tumors. Similar to previous studies (Tara et al. 2018), we found that BCOR ${ }^{\triangle \mathrm{E} 9-10}$ no longer binds RING1B via co-IP in $\mathrm{Ptch}^{+/-} ; \mathrm{Bcor}^{\Delta E 9-10}$ tumor samples, while full-length BCOR and RING1B co-IP in $P$ tch $1^{+/-}$tumor samples (Fig. 4A). We verified that PCGF1, a PRC1.1 complex member that directly interacts with the PUFD in BCOR, no longer interacts with $\mathrm{BCOR}^{\triangle \mathrm{E} 9-10}$ either (Supplemental Fig. S5A).

In the genetically engineered mouse model, $\mathrm{BCOR}^{\triangle \mathrm{E} 9-}$ 10 is missing both the PUFD, which is responsible for PCGF1 (and RING1B) binding (Wang et al. 2018), and the C-terminal ankyrin and nonankyrin repeats, which may interact with additional proteins outside of PRC1.1 (Fig. 1K), and therefore may also mediate tumor suppression independent of PRC1.1. To verify that loss of the PUFD alone, rather than the entire $\mathrm{C}$ terminus, is responsible for Igf2 activation during medulloblastoma formation, we conducted in utero electroporation (IUE) experiments to test the role of the PUFD specifically in tumorigenesis. We generated a single plasmid carrying tandem sgRNAs targeting Ptch1 (Zuckermann et al. 2015) and Bcor at exon 13 (107 nt upstream of the PUFD-coding sequence in the linker region, Supplemental Table S3) and Cre recombinase. We injected this plasmid in the fourth ventricle of E13.5 embryos that carry a single copy transgene expressing a Rosa26::Lox-Stop-Lox-Cas9-IRES-GFP 
cassette (see the Materials and Methods for details) to generate Ptch1 and Ptch1;Bcor ${ }^{\triangle P U F D}$ medulloblastomas (Supplemental Fig. S5B-E). In Ptch1;Bcor ${ }^{\triangle P U F D}$ IUE tumors, Igf2 was overexpressed compared with Ptch1 IUE control tumors (Fig. 4B), suggesting that loss of BCOR PUFD (Supplemental Table S3) aberrantly up-regulates Igf2 transcription, likely via disruption of PRC1.1 complex assembly and targeting (Wang et al. 2018).

\section{$B C O R$ is bound at the Igf2 locus and represses gene transcription through $H 2 A K 119 U b$}

To determine whether BCOR is directly bound at the Igf2 locus, we conducted BCOR ChIP in Ptch1 $1^{+/}$and Ptch $1^{+/-} ; B \operatorname{cor}^{\Delta E 9-10}$ tumor samples (see Materials and Methods for details). The BCOR antibody used was raised against a region common to $\mathrm{BCOR}$ and $\mathrm{BCOR}^{\triangle \mathrm{E} 9-10}$ (encoded within exons 6-8) and thus detects both forms in Western blot (Fig. 1L) and ChIP. Global levels of BCOR binding were reduced but not completely lost in Ptch $^{+1-}$; Bcor ${ }^{\Delta E 9-10}$ tumors compared with Ptch1 ${ }^{+/-}$tumors (yellow panels, 2803/3093 peaks, Fig. 4C; Supplemental Table S4). There was also a small set of genes with increased binding in the $P t c h 1^{+/-} ; B \operatorname{cor}^{\Delta E 9-10}$ samples (290/3093 peaks). Importantly, we also detected 3890 peaks that were unchanged between all the replicates of the two samples, suggesting that $\mathrm{BCOR}^{\triangle \mathrm{E} 9-10}$ is present.

Next, we examined the Igf2 promoter region in $P t c h 1^{+/-}$ samples, and we found that BCOR is associated with chromatin in that region (Fig. 4D). We then checked the same region in $P t c h 1^{+/-} ; B \operatorname{BcOr}{ }^{\triangle E 9-10}$ and discovered the BCOR peak is significantly reduced at the locus (Fig. 4D,E, DiffBind, $P$-adj $<0.05)$. Given that $\mathrm{BCOR}^{\triangle \mathrm{E} 9-10}$ no longer binds to the catalytic subunit of the PRC1.1 (Fig. 4A), residual $\mathrm{BCOR}^{\triangle \mathrm{E} 9-10}$ would only be capable of PRC1.1-independent functions.

If $\mathrm{BCOR}^{\triangle \mathrm{E} 9-10}$ renders the PRC1.1 nonfunctional, then H2AK119Ub levels should be reduced compared with Ptch $1^{+/-}$controls. To test this hypothesis, we performed H2AK119Ub ChIP from chromatin prepared from the same tumor samples (see the Materials and Methods for details). We found that total levels of H2AK119Ub decreased at peaks where BCOR signal is significantly decreased (red panels, Fig. 4C), indicating that the enzymatic activity of PRC1.1 complex is indeed reduced at loci with less BCOR. At the Igf2 locus, H2AK119Ub levels were significantly reduced in $\mathrm{Ptch}^{+/-} ; \mathrm{Bcor}^{\Delta E 9-10}$ tumor samples (Fig. 4D,F). We also called differential H2AK119Ub peaks between the two tumor types and then examined BCOR binding status, observing a similar effect (Supplemental Fig. S5F).

In P7 cerebellum, BCL6 and BCOR are located at promoter regions of Gli1 and Gli2 (Tiberi et al. 2014). We examined these same regions in our samples, and we found decreased BCOR binding at these sites (Supplemental Fig. S5G,H). However, H2AK119Ub levels were not significantly different at these regions, and there was no corresponding overexpression of Gli1 or Gli2 in Ptch $1^{+/-} ;$Bcor $^{\Delta E 9-10}$ tumors (Supplemental Fig. S5G,H; Supplemental Table S1). These results suggest that that the recruitment of BCOR by BCL6 is not required to maintain repression of Gli1/Gli2. The maintenance of $\mathrm{H} 2 \mathrm{AK} 119 \mathrm{Ub}$ at these loci suggests that other PRC1 complexes may compensate to prevent Gli1/ Gli2 overexpression.

Altogether, our results suggest that lack of full-length BCOR in $P$ tch $1^{+/-} ; B \operatorname{cor}^{\Delta E 9-10}$ tumors leads to direct upregulation of $I g f 2$, and this aberrant $I g f 2$ expression is closely associated with reduced H2AK119Ub and a disrupted PRC1.1 complex (Fig. 4G), enhancing the incidence of malignant transformation during tumor progression.

\section{Discussion}

\section{BCOR functions as a tumor suppressor in $\mathrm{SHH}$ medulloblastoma}

BCOR is a large, multidomain protein implicated as a tumor suppressor in many pediatric cancers (Astolfi et al. 2019). In the case of medulloblastoma, BCOR is comutated with aberrant activation of $\mathrm{SHH}$ signaling. To determine how mutations in the C-terminal domain of $B C O R$ may contribute to tumorigenesis, we used a Ptch1 loss-offunction allele together with a conditional mouse Bcor allele. This allele, $B \operatorname{cor}^{\Delta E 9-10}$, is similar to some of the human $B C O R$ mutations, where a C-terminally truncated protein, lacking the region required for interaction with PRC1.1 subunits, is predicted to be produced. Bcor ${ }^{\triangle E 9-10}$ must be at least a partial loss of function because we removed the domain critical for PRC1.1 interaction, and the levels of the truncated protein are significantly reduced in GNPs. However, we cannot exclude the possibility that the residual protein retains activity, but this activity is likely independent of PRC1.1.

It has been proposed previously that BCOR, in conjunction with BCL6 and SIRT1, represses Gli1/Gli2 (Tiberi et al. 2014). Although binding of BCOR to the Gli1/Gli2 loci is significantly reduced, Gli1/Gli2 mRNA expression is normal in $\mathrm{Ptch}^{+/-} ; B \mathrm{Cor}^{\triangle E 9-10}$ tumors, indicating that the residual activity is sufficient to repress Gli1/Gli2. Thus, our results show that the $B C O R$ tumor suppressor functions modeled here act independently of the previously described BCL6/BCOR/SIRT1 regulation of Gli1/Gli2 (Tiberi et al. 2014). Furthermore, recurrent BCL6 mutations have not been identified in SHH medulloblastoma patient samples, suggesting that BCOR and its PRC1.1-related functions may be relevant to human tumor formation.

\section{BCOR directly represses Igf2 via PRC1.1 complex- mediated H2AK119 ubiquitination}

Our results suggest that BCOR directly represses Igf2 transcription via interaction with other components of the PRC1.1 complex, which deposits H2AK119Ub repressive histone marks at the Igf2 promoter. In $P t c h 1^{+/-}$medulloblastoma, BCOR (and likely the entire PRC1.1 complex) is physically present at the Igf2 promoter, K119 on histone $\mathrm{H} 2 \mathrm{~A}$ is ubiquitylated, and subsequently $\operatorname{Ig} f 2$ is repressed (no IGF2 protein is made). In Ptch $1^{+/-} ;$Bcor $^{\Delta E 9-10}$ 
Kutscher et al.
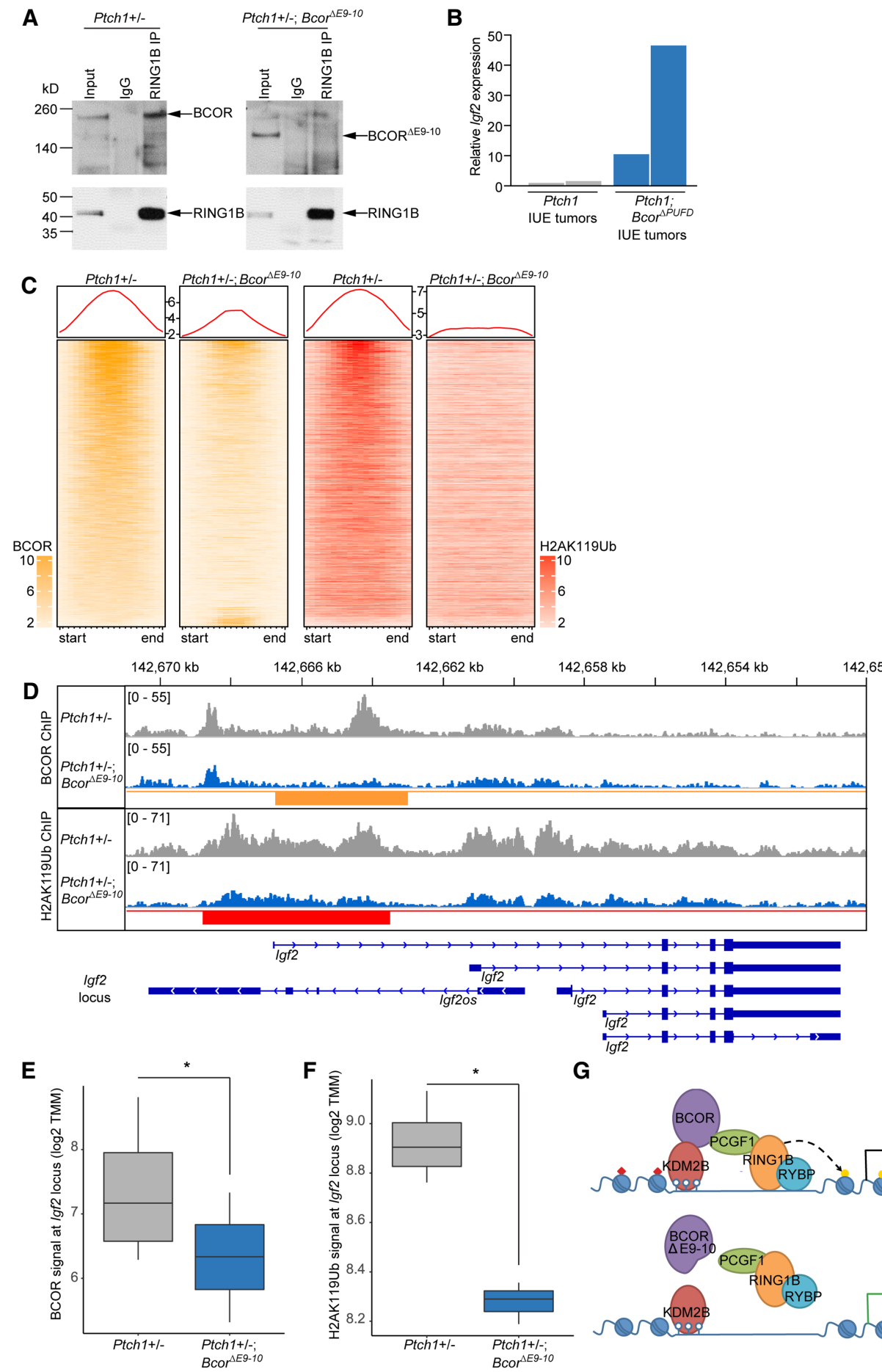

G

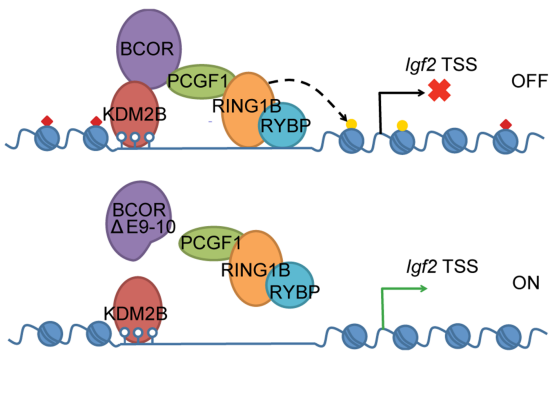

Figure 4. The PUFD of BCOR is required for Igf2 repression through H2AK119 Ubiquitination. (A) Coimmunoprecipitation of BCOR or $\mathrm{BCOR}^{\Delta \mathrm{E} 9-10}$ with RING1B in Ptch1 ${ }^{+/-}$and $P t c h 1^{+/-} ; B \operatorname{cor}{ }^{\Delta E 9-10}$ tumor cells, respectively. (IgG) Immunoglobulin G control. Five percent input. (IP) Anti-RING1B. (Top blot) Anti-BCOR. (Bottom blot) Anti-RING1B. (B) Relative mRNA expression of Igf2 in two Ptch1 CRISPR tumors (gray), and two Ptch1;Bcor ${ }^{\triangle P U F D}$ CRISPR-engineered tumors (blue). (C) Heat map of significant differentially bound peaks from BCOR chromatin immunoprecipitation (ChIP; yellow) between tumors from the indicated genotypes and the status of H2AK119Ub ChIP signals (red) within these genomic loci. $(D)$ Combined BCOR and H2AK119Ub ChIP peaks in the indicated genotypes. Significantly different peak heights represented with yellow (BCOR) and red (H2AK119Ub) bars (BCOR peaks called first). Igf2 locus is represented in blue. (E) Quantification of BCOR ChIP signal at Igf2 locus. Differential peak calling with DiffBind. $\left(*^{*} P\right.$-adj $<0.05$. $(F)$ Quantification of H2AK119Ub ChIP signal at Igf2 locus. Differential peak calling with DiffBind. (*) $P$-adj $<0.05$. (G) Model of PRC1.1 complex disruption. (Open lollipops) Unmethylated CpG; (TSS) transcriptional start site; (yellow dots) H2AK119Ub histone marks; (red diamonds) H3K27me3 histone marks. See also Supplemental Tables S3 and S4 and Supplemental Figure S5. 
medulloblastoma, however, BCOR protein levels are significantly decreased, and BCOR is no longer present at the Igf2 promoter. As a result, the PRC1.1 complex cannot be recruited, so H2AK119Ub levels are lower. Since the repressor complex is no longer present, Igf2 expression is derepressed to yield IGF2 protein. We do not yet know the mechanism by which Igf2 is activated downstream from BCOR loss, only that loss alleviates the repression. In the future, it will be interesting to investigate the mechanism(s) responsible for activating Igf2 expression, as these may be relevant for treatment across different tumor types.

While our ChIP studies were conducted in progressive murine medulloblastomas, this mechanism likely occurs in preneoplastic lesions of $P t c h 1^{+/-}$animals as well /given Igf2 up-regulation in P28 Ptch1 ${ }^{+/-} ;$Bcor $^{\Delta E 9-10}$ cerebellum). $85 \%$ of $P t c h 1^{+/-}$animals have preneoplastic lesions at P21, but only $15 \%-20 \%$ of animals develop progressive medulloblastoma (Corcoran et al. 2008; Kessler et al. 2009), suggesting that BCOR-PRC1.1 may function in preneoplastic cells to prevent aberrant Igf2 overexpression. This hypothesis is further strengthened by the fact that IGF2 is necessary for late stage $\mathrm{SHH}$ medulloblastoma progression (Hahn et al. 2000; Corcoran et al. 2008). Nevertheless, no recurrent mutations of genes that encode components of PRC1.1 other than BCOR have been identified in SHH medulloblastoma. Perhaps these mutations influence cell viability in the developing brain, potentially leading to cell death rather than tumorigenesis.

Igf2 up-regulation was detected previously in $P t c h 1^{+/-}$ advanced stage medulloblastomas, but not in $P t c h 1^{+/-}$ preneoplastic lesions (Corcoran et al. 2008). We did not detect substantial up-regulation of $\operatorname{Igf2}$ in $P t c h 1^{+/-}$tumors using in situ hybridization, but these differences may be explained by differences in methodology (DIG-labeled probes used in this study; ${ }^{35}$ S-UTP-labeled probes used in Corcoran et al. 2008), since we did see occasional low levels of Igf2 in Ptch1 $1^{+/}$advanced medulloblastomas as detected by qPCR. Still, these levels are significantly lower compared with $P$ tch $1^{+/} ; B$ cor ${ }^{\Delta 9-10}$ tumors.

If Igf2 up-regulation is indeed required to push tumorprone $P t c h 1^{+/-}$cells toward tumorigenesis, our results complement these findings. Lack of a functioning BCOR-PRC1.1 complex leads to up-regulation of Igf2 in preneoplastic lesions by P28 in Ptch1 ${ }^{+-}$animals, which then pushes these cells toward fast-growing, lethal tumors. Few molecular mechanisms of transformation of preneoplastic lesions to malignant cells have been uncovered, and the present study unveiled Igf2 overexpression in preneoplastic cells as one likely mechanism. This general mechanism explains why all Ptch $1^{+/-} ; B \operatorname{cor}^{\Delta E 9-10}$ mice, which express Igf2 early-on in preneoplastic lesions, develop tumors with significantly reduced latency and dramatically increased penetrance compared with Ptch $1^{+/-}$mice.

From our RNA-seq and Affymetrix data, we also found that other cancer signaling pathways are up-regulated in the Ptch $1^{+/}$Bcor ${ }^{\Delta E 9-10}$ tumors, including HIPPO, MAPK, and PI3K-Akt signaling (Supplemental Tables S1, S2). These additional pathways may also contribute to BCOR-driven malignancy and should be investigated in future studies. This is especially true given that in our human tumor samples, not all BCOR mutated medulloblastoma samples exhibit up-regulation of $I G F 2$, suggesting involvement of additional mechanisms not explored in the current study. It will be interesting to determine whether these oncogenic pathways cooperate with Igf2 and whether they are directly regulated by BCOR-PRC1.1.

\section{Translatability to human tumors}

We found that BCOR-PRC1.1 loss is associated with Igf2 overexpression in a mouse model of $\mathrm{SHH}$ medulloblasto$\mathrm{ma}$, and we identified three out of six cases of BCOR mutated human SHH medulloblastoma samples where IGF2 is up-regulated, suggesting that our finding may be generally applicable to some $B C O R$ mutated tumor samples. However, caution is warranted when translating our findings to human tumors. First, we did not have sample-matched mutation and expression data for all of our patient tumor samples, so the power of our human findings is low. For the six we did have, only three showed up-regulation of IGF2 compared with BCOR wild-type SHH medulloblastomas, suggesting this mechanism of tumorigenesis may be conserved only for certain mutation types.

In future studies involving human medulloblastoma samples, it will be important to fully understand the spectrum of $B C O R$ mutations and how these may influence other oncogenic pathways. For example, in one of the samples that did not show IGF2 up-regulation, BCOR carried only a point mutation (S1358Y). BCOR auto-represses itself, using its C-terminal domain (Wamstad et al. 2008). When the C-terminal domain is missing, BCOR expression increases (as seen in the MB12 sample). In S1358Y, $B C O R$ expression remains low, suggesting its C-terminal repression may still be intact. The $S 1358 Y$ point mutation is in a highly conserved amino acid, so the mode of BCORdriven tumorigenesis in this sample likely differs from our mouse model, especially since IGF2 was not up-regulated in this tumor.

\section{IGF2 overexpression may be a general feature} of BCOR-altered tumors

ITDs in the $\mathrm{C}$ terminus of BCOR have been reported not only in brain tumors, such as CNS-HGNET-BCOR tumors, but also CCSK and uterine sarcomas (Roy et al. 2015; Ueno-Yokohata et al. 2015; Mariño-Enriquez et al. 2018). Defects in the $C$ terminus of BCOR are closely associated with high expression of IGF2 in both CNSHGNET-BCOR (Vewinger et al. 2019) and CCSKs (Schuster et al. 2003), suggesting that suppression of IGF2 expression may be a general feature of BCOR function across tumor entities. Furthermore, both of the tumors exhibit aberrant activation of the SHH pathway (our studies, Paret et al. 2016), suggesting that both SHH pathway activation and $B C O R$ aberrations cooperate to activate $I G F 2$, similar to our Ptch1 $1^{+/-}$; Bcor ${ }^{\Delta E 9-10}$ mouse tumors. 
As we uncovered, Ptch1 mutated tumors and $P t c h 1^{+/}$; $B C{ }^{\Delta E 9-10}$ tumors have different tumorigenic properties, including penetrance, latency, and mechanism of tumorigenesis. If this association holds true across tumor types, patients with specific $B C O R$ and $\mathrm{SHH}$-related mutations may benefit from different treatment strategies compared with SHH medulloblastomas lacking BCOR mutations. More mechanistic studies are warranted to determine a possible link between the type of $B C O R$ mutation and IGF2 up-regulation. Our results also demonstrate the need to investigate the cooperating mutations for each subtype of medulloblastoma, and by extension, other tumor subtypes, to understand how these mutations contribute to malignant phenotypes.

\section{Materials and methods}

Animals

$B c o r$ conditional knockout mice were generated by breeding of $B{ }^{f}{ }^{\text {fl/fl }}$ (loxP sites flanking exons 9 and 10) (Hamline et al. 2020) with Atoh1-Cre (JAX 011104) and Ptch1 heterozygous mice (Goodrich et al. 1997). Genotyping primer sequences are available in Supplemental Table S5. All mice were bred on a C57BL/6N background. For tumor studies, only homozygous $B$ cor $^{f l / f 1}$ females or $B$ cor $^{f 1 / y}$ males were used and referred to as $B C o r^{\Delta E 9-10}$. Of note, $B \operatorname{cor}^{f 1 /+}$ females developed medulloblastoma at similar rates. For the in utero electroporation experiments, male Rosa26-CAG-LSL-Cas9-P2A-EGFP (JAX 024857) mice were crossed to CD-1 female animals purchased from Janvier and checked daily for vaginal plug. For transplantation experiments, Nod-scid IL2R $\gamma^{\text {null }}$ (NSG; JAX 005557) were used. All animal experiments for this study were conducted according to the animal welfare regulations approved by the Animal Care and Use Committee of the National Institute of Neuroscience (NCNP) Japan (approval no. 2019028R2), and the responsible authorities in Baden-Württemberg, Germany (Regierungspräsidium Karlsruhe, approval nos. G-182/13, G-48/14, G-64/14, G-29/18, and G-23/19).

In utero electroporation In utero electroporation was performed as described previously (Zuckermann et al. 2015). For these experiments, we used a single px330 plasmid (Addgene \#42230) modified to carry two sgRNA cassettes, and Cre recombinase coding sequence was used in place of Cas9. This plasmid together with a luciferase-encoding plasmid was injected into the fourth ventricle of E13.5 embryos and electroporated $(30 \mathrm{~V}, 50 \mathrm{msec}$ on, 950 msec off, five pulses). Positive pups were identified by luciferase signal at $\mathrm{P} 7$ and animals were monitored daily for the first neurological signs of medulloblastoma formation.

Orthotopic transplantation For retransplantation experiments, $8 \times 10^{5}$ freshly isolated, purified tumor cells from $\mathrm{Ptch}^{+/-}$or $\operatorname{Ptch}^{+/-} ; B \operatorname{cor}^{\Delta E 9-10}$ animals were retransplanted to the cerebellum of NSG mice, according to published standard operating procedures (Brabetz et al. 2018), and animals were monitored daily for signs of tumor growth.

For Igf2 overexpression studies, retroviruses were freshly generated as described previously (Kawauchi et al. 2012). P7 GNPs isolated from $P t c h 1^{+/-}$mice were cultured in vitro and transduced with MSCV-based retroviruses carrying Igf2-IRES-eGFP, MycnIRES-eGFP (positive control), or eGFP only (negative control). Two days later, infected GNPs were harvested and transplanted to NSG mice $\left(1 \times 10^{6}\right.$ cells per mouse $)$ in the same manner as above.

\section{Plasmids}

A single px330 plasmid modified to carry Cre instead of Cas9 was generated to carry tandem cassettes for sgRNAs against Ptch1 (CTGGCCGGAAAGCGCCGCTG) and Bcor (ATAGAACTCC CAAGCGCCGC), using ASAP cloning protocol (Zuckermann et al. 2018). For Ptch1-only targeting construct, the sgRNA for $B c o r$ was replaced with a control sgRNA (GCGACCAATACGC GAACGTC). For MSCV-based plasmids, the coding region for Igf2, Mycn, or empty vector was cloned using BamHI and XhoI sites. For co-IP plasmids in HEK293T cells, full-length Bcor cDNA or truncated $B c r^{\triangle E 9-10}$ cDNA was cloned with an N-terminal HA tag into pCAG vector using InFusion (Takara). pCMV-SPORT 6.1 with mouse Bcl6 cDNA was purchased from Horizon Discovery (clone ID 6309948).

\section{In situ hybridization}

In situ hybridization (ISH) was performed as described previously (Kawauchi et al. 2006). Plasmids containing the coding region of Bcor (clone ID 6412868, Horizon Discovery), Igf2 (clone ID 30013295, Horizon Discovery), and Ccnd2 (5716186, Horizon Discovery) were linearized with XbaI, EcoRI, and AccI, respectively, and used to generate antisense RNA probes (DIG RNA labeling kit, Roche). Probes were hybridized on $10-\mu \mathrm{m}$-thick cryosections of the cerebellum or tumor samples of interest and counterstained with methyl green (Sigma). For expression pattern analysis, we checked three independent cerebella and all ISH results reported in this study were consistent in all three samples.

\section{Histopathology and Immunostaining}

For histopathology, samples of murine medulloblastomas (three tumors per genotype) were formalin-fixed, paraffin-embedded, and sectioned at $5 \mu \mathrm{m}$. The sections were stained with hematoxylin and eosin $(\mathrm{H} \& \mathrm{E})$, and histological classifications were performed blinded to genotype.

Immunostaining was performed as described previously (Pajtler et al. 2019). Brains were harvested, fixed overnight in $4 \%$ paraformaldehyde (PFA) at $4^{\circ} \mathrm{C}$, soaked in $30 \%$ sucrose in PBS overnight, and embedded in optimal cutting temperature (OCT) compound. Frozen $10-\mu \mathrm{m}$-thick sections were collected on Fisher Superfrost Plus slides using a cryostat. Sections were blocked for $30 \mathrm{~min}$ at room temperature with $10 \%$ normal donkey serum and incubated with primary antibody overnight at $4^{\circ} \mathrm{C}$. After washing with PBST, sections were incubated with appropriate fluorescence secondary antibodies for $1 \mathrm{~h}$ at room temperature.

In the case of BCOR immunostaining, P7 brains were fixed at $1 \mathrm{~h}$ in 4\% PFA and P28 brains were perfused with 4\% PFA for 1 $\mathrm{h}$, prior to soaking in $20 \%$ sucrose overnight. Sections were cut at $16 \mu \mathrm{m}$ and permeabilized in $1 \% \mathrm{BSA}+0.4 \%$ Triton/PBS for $1 \mathrm{~h}$ at room temperature. Anti-BCOR antibody was incubated overnight at room temperature, and sections were washed with PBS prior to secondary antibody incubation.

Slides were mounted in ProLong Gold mountant (Invitrogen). Antibodies used are listed in Supplemental Table S5. Samples were counterstained with $300 \mathrm{nM}$ DAPI. For quantification, we counted the number of positive cells in four $100-\mu \mathrm{m} \times 100-\mu \mathrm{m}$ regions in three independent cerebella. 


\section{Quantitative PCR ( $q P C R$ )}

GNP isolation was performed as previously described (Kawauchi et al. 2012). For in vitro studies, five million P7 GNPs were plated per well in a six-well plate and cultured for $48 \mathrm{~h}$ in the presence or absence of $200 \mathrm{nM}$ smoothened agonist (Merck). RNA was extracted and cDNA was generated with SuperScript II kit (Invitrogen), according to the manufacturer's instructions. RNA and cDNA from tumor samples was generated similarly. Relative gene expression was compared with Gapdh in all experiments except Figures $3 \mathrm{P}$ and $4 \mathrm{~B}$, which was compared with the geometric mean of Hrpt1, Rpl27, and Rer1, and Supplemental Figure S1D, which was compared with $18 \mathrm{~s}$ rRNA. All primer sequences are listed in Supplemental Table S5.

\section{Western blotting}

Protein was extracted from tissue or cells by addition of RIPA buffer (Sigma Aldrich) including complete protease inhibitor cocktail tablets (Roche) according to the manufacturer's instruction. Tissue was homogenized using the Tissue Master 125 homogenizer (OMNI International) or sonication after adding lysis buffer directly to the frozen sample. Cell lysates were vortexed three to four times every $10 \mathrm{~min}$ for a total of $30 \mathrm{~min}$ and were kept on ice in between. Lysates were centrifuged at 17,000 for $30 \mathrm{~min}$ at $4^{\circ} \mathrm{C}$. Protein concentration of cell lysates was measured using Pierce BCA protein assay (Thermo Fisher Scientific), following the supplier's instructions. Primary antibodies are listed in Supplemental Table S5.

\section{Co-IP}

For interaction tests between RING1B and BCOR-FL / BCOR ${ }^{\triangle \mathrm{E} 9-10}$ in mouse tumor samples, tumor cells were lysed in LB200 buffer with sonication. Anti-RING1B antibody was bound to IgG beads (1:500; Active Motif 39663) for $1 \mathrm{~h}$ at room temperature before applying $500 \mu \mathrm{g}$ of the total cell lysate. Proteins were allowed to bind for $4 \mathrm{~h}$ at room temperature while rotating. After three wash steps with LB200 at room temperature, bound proteins were eluted in $2 \times$ SDS Laemmli buffer. Total elution was loaded on $4 \%-12 \%$ bis-Tris gradient gel (NuPAGE, Thermo Fisher Scientific) for downstream Western blotting.

\section{$X$-gal whole-mount staining}

X-gal staining was performed according to standard protocol. Brains were fixed in fresh $4 \%$ PFA / PBS for $1 \mathrm{~h}$ on ice, rinsed in Rinse Buffer (100 mM sodium phosphate at $\mathrm{pH} 7.3,2 \mathrm{mM} \mathrm{MgCl}_{2}$, $0.01 \%$ sodium deoxycholate, $0.02 \%[\mathrm{w} / \mathrm{v}] \mathrm{NP}-40$ ) three times for $10 \mathrm{~min}$, followed by X-gal staining solution $(5 \mathrm{mM}$ potassium ferricyanide, $5 \mathrm{mM}$ potassium ferrocyanide, $1 \mathrm{mg} / \mathrm{mL} \mathrm{X-gal} \mathrm{in} \mathrm{DMF)}$ overnight at $37^{\circ} \mathrm{C}$. After, brains were placed in $10 \%$ formalin and the number of preneoplastic lesions per cerebellum was quantified on a stereomicroscope ( $N=3$ per genotype).

Human tumor data analysis

The presence of $B C O R$ mutations and indels was integrated from our previous medulloblastoma studies (Kool et al. 2014; Northcott et al. 2017; Waszak et al. 2020). These mutations were mapped onto NP_001116857.1. Where available, the IGF2 gene expression was checked using RNA-seq data and Affymetrix data. Additionally, the BCOR somatic indel in sample MB12 was confirmed via RNA-seq alignment data. However, we only detected $2 \%$ of reads with the missense mutation in MB234.
FPKM gene expression values from selected tumor samples from INFORM were compared across entities $(B C O R$-WT group 3/4 medulloblastoma, BCOR-WT SHH medulloblastoma, CNSHGNET-BCOR, and CCSK).

Mouse model gene expression analysis

RNA extraction Tissue was homogenized using the Tissue Master 125 homogenizer (OMNI International) and/or QIAshredder (Qiagen). RNA was extracted with RNeasy mini kit (Qiagen) following the supplier's instructions. For measurement of RNA quantity, ND1000 Spectrophotometer (NanoDrop Technologies) and/or Agilent 2100 Bioanalyzer was used. RNA-seq libraries were processed and sequenced on a HiSeq V4 (SR 50) by the DKFZ Genomics Core Facility.

RNA-seq analysis Transcriptome profiling (reads alignment to mm10 reference, quality control and gene expression counts computation) was performed using RNA sequencing data from Ptch1 ${ }^{+-}(N=3)$ and $P t c h 1^{+/-} ; B \operatorname{cor}^{\Delta E 9-10}(N=3)$ medulloblastoma tumor models as described previously (Pajtler et al. 2019). Differentially expressed genes were detected using DESeq2 R package with applied limits: minimum $P$-adj 0.05 , minimum $\log _{2}$ FC 0.5 .

Additional extended transcriptome profiling from $\operatorname{Ptch}^{+/-}(N=$ 5) and $\operatorname{Ptch}^{+/-} ; \mathrm{Bcor}^{\Delta E 9-10}(\mathrm{~N}=4)$ medulloblastoma SHH tumor models was performed with Affymetrix microarray 430v2. The differentially expressed genes were detected using Limma package (Ritchie et al. 2015) and sorted using p-adj limit for the top 1000 selection. DAVID gene ontology analysis was applied to the RNA-seq and Affymetrix data with a focus on biological processes and KEGG pathways.

The mouse tumor models were compared with the ICGC medulloblastoma RNA-seq cohort $(n=170)$. Ortholog selection was performed using the biomart $\mathrm{R}$ package via corresponding Ensembl database annotation. After batch effect adjustment with Combat (Leek et al. 2012), unsupervised clustering was performed with ward.D2 method on the top 500 most highly variable genes. To understand how the mouse models correspond to the human tumors, the ICGC cohort was restricted to the SHH subgroup only, and principal component analysis was performed on the combined batch effect adjusted gene expression counts, using the top 100 differentially expressed genes between $P t c h 1^{+/-}$and Ptch1 ${ }^{+/-} ; B \operatorname{cor}^{\triangle E 9-10}$ tumors as the main input.

ChIP sequencing data analysis ChIP of H2AK1 19Ub /Cell Signaling Technologies) and BCOR (Vivian Bardwell Laboratory, University of Minnesota, anti-BCOR, RRID:AB_2716801) for Ptch1 ${ }^{+/}$; $B$ cor $^{\triangle E 9-10}$ and $P t c h 1^{+/-}$tumor samples was performed by Active, Motif. One sample of $P t c h 1^{+/-} ; B \operatorname{cor}^{\Delta E 9-10}$ was discarded for outlier levels of Igf2 revealed by RNA-seq after ChIP analysis. The sequencing reads representing H2AK119Ub and BCOR obtained from $P t c h 1^{+/-}(N=4)$ and $\operatorname{Ptch}^{1+/-} ; B \operatorname{cor}^{\Delta E 9-10}(N=3)$ tumor model samples were aligned to the $\mathrm{mm} 10$ and Drosophila spike-in references using bwa 0.6.2. Next, the general quality control and read alignments statistics collection was performed using Qualimap v2.2 toolkit mode BAM QC (Okonechnikov et al. 2016). The coefficients from Drosophila spike-ins were computed based on the alignment statistics and applied to adjust to the reads distribution per sample. For peak calling Macs 1.4 tool (Zhang et al. 2008) was applied with corresponding background included as input and using $P$-value limit $1 \times 10^{-9}$. Differential peaks were detected using DiffBind R package (Ross-Innes et al. 2012) with minimum $P$-adj of 0.05 . 


\section{Acknowledgments}

We thank Annarita Patrizi, Ana Banito, Mija Blattner-Johnson, and David T.W. Jones for their scientific input and discussions. Support by the GPCF Antibody Unit (Dr. Ilse Hofmann), DKFZ Light Microscopy Facility, and DKFZ Genomics Facility is gratefully acknowledged. This work was supported by a Deutsche Forschungsgemeinschaft grant (KA4472/1-1) and a grant from the Japan Agency for Medical Research and Development (AMED; JP20ck0106534h0001) to D.K., a Heinrich F.C. Behr Stipendium to N.V.B., and National Institutes of Health grants 5R01CA071540 and R01HD084459 to V.J.B.

Author contributions: N.V.B., P.A.N., and D.K. conceived the project. L.M.K., N.V.B., J.C., P.B.G.S., M.V., S.v.R., L.S., B.S., R.S., and D.K. conducted in vitro experiments. L.M.K., N.V.B., J.C., P.B.G.S., N.M., and D.K. performed animal experiments. K.O., B.L.G., and K.S.S. performed computational analysis. B.A.O. and A.K. performed histopathological analysis. M.D.G., V.J.B., S.M.P., A.L.M., O.A., M.K., M.H., K.v.H., N.G., and G.F. provided biomaterials and resources. L.M.K. wrote the original draft of the manuscript. L.M.K., K.O., N.V.B., M.D.G., V.J.B., S.M.P., P.A.N., and D.K. edited the manuscript. All authors reviewed and approved the manuscript. S.M.P., P.A.N., and D.K. supervised the project.

\section{References}

Astolfi A, Melchionda F, Perotti D, Fois M, Indio V, Urbini M, Genovese CG, Collini P, Salfi N, Nantron M, et al. 2015. Whole transcriptome sequencing identifies BCOR internal tandem duplication as a common feature of clear cell sarcoma of the kidney. Oncotarget 6: 40934-40939. doi:10.18632/onco target.5882

Astolfi A, Fiore M, Melchionda F, Indio V, Bertuccio SN, Pession A. 2019. BCOR involvement in cancer. Epigenomics 11: 835855. doi:10.2217/epi-2018-0195

Brabetz S, Leary SES, Gröbner SN, Nakamoto MW, Seker-Cin H, Girard EJ, Cole B, Strand AD, Bloom KL, Hovestadt V, et al. 2018. A biobank of patient-derived pediatric brain tumor models. Nat Med 24: 1752-1761. doi:10.1038/s41591-018-0207-3

Cao Q, Gearhart MD, Gery S, Shojaee S, Yang H, Sun H, Lin D-C, Bai J-W, Mead M, Zhao Z, et al. 2016. BCOR regulates myeloid cell proliferation and differentiation. Leukemia 30: 11551165. doi:10.1038/leu.2016.2

Corcoran RB, Bachar Raveh T, Barakat MT, Lee EY, Scott MP. 2008. Insulin-like growth factor 2 is required for progression to advanced medulloblastoma in patched1 heterozygous mice. Cancer Res 68: 8788-8795. doi:10.1158/0008-5472 .CAN-08-2135

Gearhart MD, Corcoran CM, Wamstad JA, Bardwell VJ. 2006. Polycomb group and SCF ubiquitin ligases are found in a novel BCOR complex that is recruited to BCL6 targets. Mol Cell Biol 26: 6880-6889. doi:10.1128/MCB.00630-06

Ghetu AF, Corcoran CM, Cerchietti L, Bardwell VI, Melnick A, Privé GG. 2008. Structure of a BCOR corepressor peptide in complex with the BCL6 BTB domain dimer. Mol Cell 29: 384-391. doi:10.1016/j.molcel.2007.12.026

Goodrich LV, Milenković L, Higgins KM, Scott MP. 1997. Altered neural cell fates and medulloblastoma in mouse patched mutants. Science 277: 1109-1113. doi:10.1126/science.277.5329 .1109

Grossmann V, Tiacci E, Holmes AB, Kohlmann A, Martelli MP, Kern W, Spanhol-Rosseto A, Klein H-U, Dugas M, Schindela $S$, et al. 2011. Whole-exome sequencing identifies somatic mutations of BCOR in acute myeloid leukemia with normal karyotype. Blood 118: 6153-6163. doi:10.1182/blood-201107-365320

Hahn H, Wicking C, Zaphiropoulous PG, Gailani MR, Shanley S, Chidambaram A, Vorechovsky I, Holmberg E, Unden AB, Gillies S, et al. 1996. Mutations of the human homolog of Drosophila patched in the nevoid basal cell carcinoma syndrome. Cell 85: 841-851. doi:10.1016/S0092-8674(00)81268-4

Hahn H, Wojnowski L, Specht K, Kappler R, Calzada-Wack J, Potter D, Zimmer A, Müller U, Samson E, Quintanilla-Martinez L, et al. 2000. Patched target Igf2 is indispensable for the formation of medulloblastoma and rhabdomyosarcoma. I Biol Chem 275: 28341-28344. doi:10.1074/jbc.C000352200

Hamline MY, Corcoran CM, Wamstad JA, Miletich I, Feng J, Lohr JL, Hemberger M, Sharpe PT, Gearhart MD, Bardwell VJ. 2020. OFCD syndrome and extraembryonic defects are revealed by conditional mutation of the polycomb-group repressive complex 1.1 (PRC1.1) gene Bcor. Dev Biol doi:10.1016/j.ydbio .2020 .06 .013

Huynh KD, Fischle W, Verdin E, Bardwell VJ. 2000. BCoR, a novel corepressor involved in BCL-6 repression. Genes Dev 14: 1810-1823.

Johnson RL, Rothman AL, Xie J, Goodrich LV, Bare JW, Bonifas JM, Quinn AG, Myers RM, Cox DR, Epstein EH, et al. 1996. Human homolog of patched, a candidate gene for the basal cell nevus syndrome. Science 272: 1668-1671. doi:10.1126/sci ence.272.5268.1668

Junco SE, Wang R, Gaipa JC, Taylor AB, Schirf V, Gearhart MD, Bardwell VI, Demeler B, Hart PJ, Kim CA. 2013. Structure of the polycomb group protein PCGF1 in complex with BCOR reveals basis for binding selectivity of PCGF homologs. Structure 21: 665-671. doi:10.1016/j.str.2013.02.013

Kawauchi D, Taniguchi H, Watanabe H, Saito T, Murakami F. 2006. Direct visualization of nucleogenesis by precerebellar neurons: involvement of ventricle-directed, radial fibre-associated migration. Development 133: 1113-1123. doi:10 $.1242 /$ dev.02283

Kawauchi D, Robinson G, Uziel T, Gibson P, Rehg J, Gao C, Finkelstein D, Qu C, Pounds S, Ellison DW, et al. 2012. A mouse model of the most aggressive subgroup of human medulloblastoma. Cancer Cell 21: 168-180. doi:10.1016/j.ccr.2011.12.023

Kenney AM, Cole MD, Rowitch DH. 2003. Nmyc upregulation by sonic hedgehog signaling promotes proliferation in developing cerebellar granule neuron precursors. Development 130: 15-28. doi:10.1242/dev.00182

Kessler JD, Hasegawa H, Brun SN, Emmenegger BA, Yang Z-J, Dutton JW, Wang F, Wechsler-Reya RJ. 2009. N-myc alters the fate of preneoplastic cells in a mouse model of medulloblastoma. Genes Dev 23: 157-170. doi:10.1101/gad.1759909

Kooi IE, Mol BM, Massink MPG, Ameziane N, Meijers-Heijboer $\mathrm{H}$, Dommering CJ, van Mil SE, de Vries Y, van der Hout AH, Kaspers GJL, et al. 2016. Somatic genomic alterations in retinoblastoma beyond RB1 are rare and limited to copy number changes. Sci Rep 6: 25264. doi:10.1038/srep25264

Kool M, Jones DTW, Jäger N, Northcott PA, Pugh TJ, Hovestadt V, Piro RM, Esparza LA, Markant SL, Remke M, et al. 2014. Genome sequencing of SHH medulloblastoma predicts genotype-related response to smoothened inhibition. Cancer Cell 25: 393-405. doi:10.1016/j.ccr.2014.02.004

Lee EY, Ji H, Ouyang Z, Zhou B, Ma W, Vokes SA, McMahon AP, Wong WH, Scott MP. 2010. Hedgehog pathway-regulated gene networks in cerebellum development and tumorigenesis. Proc Natl Acad Sci 107: 9736-9741. doi:10.1073/pnas.1004602107

Leek JT, Johnson WE, Parker HS, Jaffe AE, Storey JD. 2012. The sva package for removing batch effects and other unwanted 
variation in high-throughput experiments. Bioinformatics 28: 882-883. doi:10.1093/bioinformatics/bts034

Mackay A, Burford A, Carvalho D, Izquierdo E, Fazal-Salom J, Taylor KR, Bjerke L, Clarke M, Vinci M, Nandhabalan M, et al. 2017. Integrated molecular meta-analysis of 1,000 pediatric high-grade and diffuse intrinsic pontine glioma. Cancer Cell 32: 520-537.e5. doi:10.1016/j.ccell.2017.08.017

Mariño-Enriquez A, Lauria A, Przybyl J, Ng TL, Kowalewska M, Debiec-Rychter M, Ganesan R, Sumathi V, George S, McCluggage WG, et al. 2018. BCOR internal tandem duplication in high-grade uterine sarcomas. Am J Surg Pathol 42: 335-341. doi:10.1097/PAS.0000000000000993

Northcott PA, Dubuc AM, Pfister S, Taylor MD. 2012. Molecular subgroups of medulloblastoma. Expert Rev Neurother 12: 871-884. doi:10.1586/ern.12.66

Northcott PA, Buchhalter I, Morrissy AS, Hovestadt V, Weischenfeldt J, Ehrenberger T, Gröbner S, Segura-Wang M, Zichner T, Rudneva VA, et al. 2017. The whole-genome landscape of medulloblastoma subtypes. Nature 547: 311-317. doi:10 $.1038 /$ nature22973

Oki S, Ohta T, Shioi G, Hatanaka H, Ogasawara O, Okuda Y, Kawaji H, Nakaki R, Sese J, Meno C. 2018. ChIP-Atlas: a data-mining suite powered by full integration of public ChIP-seq data. EMBO Rep 19: e46255.

Okonechnikov K, Conesa A, García-Alcalde F. 2016. Qualimap 2: advanced multi-sample quality control for high-throughput sequencing data. Bioinformatics 32: 292-294.

Oliver TG, Read T-A, Kessler JD, Mehmeti A, Wells JF, Huynh TTT, Lin SM, Wechsler-Reya RJ. 2005. Loss of patched and disruption of granule cell development in a pre-neoplastic stage of medulloblastoma. Development 132: 2425-2439. doi:10.1242/dev.01793

Pajtler KW, Wei Y, Okonechnikov K, Silva PBG, Vouri M, Zhang L, Brabetz S, Sieber L, Gulley M, Mauermann M, et al. 2019. YAP1 subgroup supratentorial ependymoma requires TEAD and nuclear factor I-mediated transcriptional programmes for tumorigenesis. Nat Commun 10: 3914-3916. doi:10 .1038/s41467-019-11884-5

Paret C, Theruvath J, Russo A, Kron B, Malki El K, Lehmann N, Wingerter A, Neu MA, Gerhold-Ay A, Wagner W, et al. 2016. Activation of the basal cell carcinoma pathway in a patient with CNS HGNET-BCOR diagnosis: consequences for personalized targeted therapy. Oncotarget 7: 83378-83391. doi:10.18632/oncotarget.13092

Ritchie ME, Phipson B, Wu D, Hu Y, Law CW, Shi W, Smyth GK. 2015. limma powers differential expression analyses for RNAsequencing and microarray studies. Nucleic Acids Res 43: e47. doi:10.1093/nar/gkv007

Ross-Innes CS, Stark R, Teschendorff AE, Holmes KA, Ali HR, Dunning MJ, Brown GD, Gojis O, Ellis IO, Green AR, et al. 2012. Differential oestrogen receptor binding is associated with clinical outcome in breast cancer. Nature 481: 389393. doi:10.1038/nature 10730

Roy A, Kumar V, Zorman B, Fang E, Haines KM, Doddapaneni H, Hampton OA, White S, Bavle AA, Patel NR, et al. 2015. Recurrent internal tandem duplications of BCOR in clear cell sarcoma of the kidney. Nat Commun 6: 8891-8897. doi:10 $.1038 /$ ncomms 9891

Santiago T, Clay MR, Allen SJ, Orr BA. 2017. Recurrent BCOR internal tandem duplication and BCOR or BCL6 expression distinguish primitive myxoid mesenchymal tumor of infancy from congenital infantile fibrosarcoma. Mod Pathol 30: 884891. doi:10.1038/modpathol.2017.12

Schüller U, Heine VM, Mao J, Kho AT, Dillon AK, Han Y-G, Huillard E, Sun T, Ligon AH, Qian Y, et al. 2008. Acquisition of granule neuron precursor identity is a critical determinant of progenitor cell competence to form Shh-induced medulloblastoma. Cancer Cell 14: 123-134. doi:10.1016/j.ccr.2008 .07 .005

Schuster AE, Schneider DT, Fritsch MK, Grundy P, Perlman EJ. 2003. Genetic and genetic expression analyses of clear cell sarcoma of the kidney. Lab Invest 83: 1293-1299. doi:10.1097/01 .LAB.0000087850.69363.59

Sturm D, Orr BA, Toprak UH, Hovestadt V, Jones DTW, Capper D, Sill M, Buchhalter I, Northcott PA, Leis I, et al. 2016. New brain tumor entities emerge from molecular classification of CNS-PNETs. Cell 164: 1060-1072. doi:10.1016/j.cell .2016 .01 .015

Tamayo-Orrego L, Charron F. 2019. Recent advances in SHH medulloblastoma progression: tumor suppressor mechanisms and the tumor microenvironment. F1000Res 8: 1823. doi:10 $.12688 /$ f1000research.20013.1

Tamayo-Orrego L, Wu C-L, Bouchard N, Khedher A, Swikert SM, Remke M, Skowron P, Taylor MD, Charron F. 2016. Evasion of cell senescence leads to medulloblastoma progression. Cell Rep 14: 2925-2937. doi:10.1016/j.celrep.2016.02.061

Tara S, Isshiki Y, Nakajima-Takagi Y, Oshima M, Aoyama K, Tanaka T, Shinoda D, Koide S, Saraya A, Miyagi S, et al. 2018. Bcor insufficiency promotes initiation and progression of myelodysplastic syndrome. Blood 132: 2470-2483. doi:10 $.1182 /$ blood-2018-01-827964

Tiberi L, Bonnefont J, van den Ameele J, Le Bon S-D, Herpoel A, Bilheu A, Baron BW, Vanderhaeghen P. 2014. A BCL6/ BCOR/SIRT1 complex triggers neurogenesis and suppresses medulloblastoma by repressing Sonic Hedgehog signaling. Cancer Cell 26: 797-812. doi:10.1016/j.ccell.2014.10.021

Udaka YT, Packer RJ. 2018. Pediatric brain tumors. Neurol Clin 36: 533-556. doi:10.1016/j.ncl.2018.04.009

Ueno-Yokohata H, Okita H, Nakasato K, Akimoto S, Hata J-I, Koshinaga T, Fukuzawa M, Kiyokawa N. 2015. Consistent in-frame internal tandem duplications of BCOR characterize clear cell sarcoma of the kidney. Nat Genet 47: 861-863. doi:10.1038/ng.3338

Vewinger N, Huprich S, Seidmann L, Russo A, Alt F, Bender H, Sommer C, Samuel D, Lehmann N, Backes N, et al. 2019. IGF1R is a potential new therapeutic target for HGNETBCOR brain tumor patients. Int I Mol Sci 20: 3027. doi:10 .3390/ijms20123027

Vogelstein B, Kinzler KW. 1993. The multistep nature of cancer. Trends Genet 9: 138-141. doi:10.1016/0168-9525(93)90209-Z

Wamstad JA, Bardwell VJ. 2007. Characterization of Bcor expression in mouse development. Gene Expr Patterns 7: 550-557. doi:10.1016/j.modgep.2007.01.006

Wamstad JA, Corcoran CM, Keating AM, Bardwell VJ. 2008. Role of the transcriptional corepressor Bcor in embryonic stem cell differentiation and early embryonic development. PLoS One 3: e2814. doi:10.1371/journal.pone.0002814

Wang H, Wang L, Erdjument-Bromage H, Vidal M, Tempst P, Jones RS, Zhang Y. 2004. Role of histone H2A ubiquitination in Polycomb silencing. Nature 431: 873-878. doi:10.1038/ nature02985

Wang Z, Gearhart MD, Lee Y-W, Kumar I, Ramazanov B, Zhang Y, Hernandez C, Lu AY, Neuenkirchen N, Deng J, et al. 2018. A non-canonical BCOR-PRC1.1 complex represses differentiation programs in human ESCs. Cell Stem Cell 22: 235251.e9. doi:10.1016/j.stem.2017.12.002

Waszak SM, Robinson GW, Gudenas BL, Smith KS, Forget A, Kojic M, Garcia-Lopez J, Hadley J, Hamilton KV, Indersie E, et al. 2020. Germline elongator mutations in sonic hedgehog 
Kutscher et al.

medulloblastoma. Nature 580: 396-401. doi:10.1038/s41586020-2164-5

Wong MK, Ng CCY, Kuick CH, Aw SJ, Rajasegaran V, Lim JQ, Sudhanshi J, Loh E, Yin M, Ma J, et al. 2018. Clear cell sarcomas of the kidney are characterised by BCOR gene abnormalities, including Exon 15 internal tandem duplications and BCOR-CCNB3 gene fusion. Histopathology 72: 320-329. doi:10.1111/his.13366

Worst BC, van Tilburg CM, Balasubramanian GP, Fiesel P, Witt R, Freitag A, Boudalil M, Previti C, Wolf S, Schmidt S, et al. 2016. Next-generation personalised medicine for high-risk paediatric cancer patients-the INFORM pilot study. Eur $J$ Cancer 65: 91-101. doi:10.1016/j.ejca.2016.06.009

Yang Z-J, Ellis T, Markant SL, Read T-A, Kessler JD, Bourboulas M, Schüller U, Machold R, Fishell G, Rowitch DH, et al. 2008. Medulloblastoma can be initiated by deletion of patched in lineage-restricted progenitors or stem cells. Cancer Cell 14: 135-145. doi:10.1016/j.ccr.2008.07.003

Zhang Y, Liu T, Meyer CA, Eeckhoute J, Johnson DS, Bernstein BE, Nussbaum C, Myers RM, Brown M, Li W, et al. 2008. Model-based analysis of ChIP-seq (MACS). Genome Biol 9: R137R139. doi:10.1186/gb-2008-9-9-r137

Zuckermann M, Hovestadt V, Knobbe-Thomsen CB, Zapatka M, Northcott PA, Schramm K, Belic J, Jones DTW, Tschida B, Moriarity B, et al. 2015. Somatic CRISPR/Cas9mediated tumour suppressor disruption enables versatile brain tumour modelling. Nat Commun 6: 7391. doi:10.1038/ ncomms 8391

Zuckermann M, Hlevnjak M, Yazdanparast H, Zapatka M, Jones DTW, Lichter P, Gronych J. 2018. A novel cloning strategy for one-step assembly of multiplex CRISPR vectors. Sci Rep 8: 17499. doi:10.1038/s41598-018-35727-3 


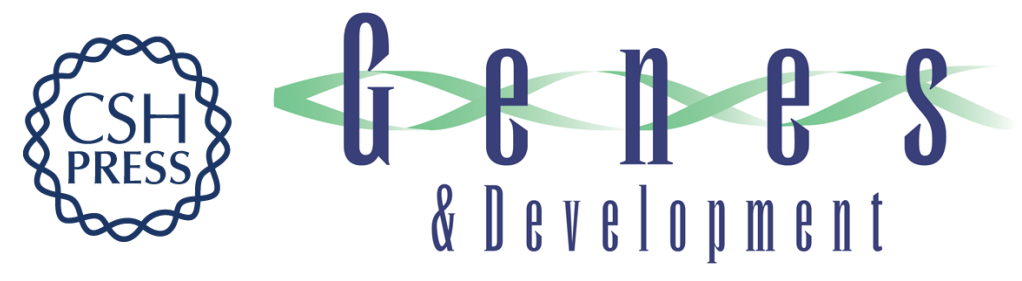

\section{Functional loss of a noncanonical BCOR-PRC1.1 complex accelerates SHH-driven medulloblastoma formation}

Lena M. Kutscher, Konstantin Okonechnikov, Nadja V. Batora, et al.

Genes Dev. 2020, 34: originally published online August 20, 2020

Access the most recent version at doi:10.1101/gad.337584.120

\section{Supplemental http://genesdev.cshlp.org/content/suppl/2020/08/19/gad.337584.120.DC1 Material}

References This article cites 58 articles, 14 of which can be accessed free at:

http://genesdev.cshlp.org/content/34/17-18/1161.full.html\#ref-list-1

Creative This article is distributed exclusively by Cold Spring Harbor Laboratory Press for the first

Commons six months after the full-issue publication date (see

License http://genesdev.cshlp.org/site/misc/terms.xhtml). After six months, it is available under a Creative Commons License (Attribution-NonCommercial 4.0 International) as described at http://creativecommons.org/licenses/by-nc/4.0/.

Email Alerting Receive free email alerts when new articles cite this article - sign up in the box at the top Service right corner of the article or click here.

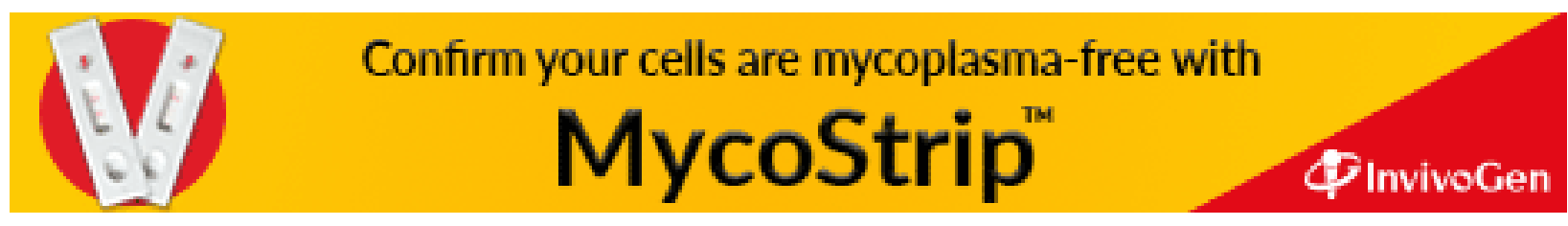

